\title{
Divergence Property of Formal Solutions for Singular First Order Linear Partial Differential Equations
}

By

\author{
Masaki HiBINo*
}

\begin{abstract}
This paper is concerned with the study of the convergence and the divergence of formal power series solutions of the following first order singular linear partial differential equation with holomorphic coefficients at the origin:

$$
P(x, D) u(x)=\sum_{l=1}^{d} a_{l}(x) D_{l} u(x)+b(x) u(x)=f(x)
$$

with $f(x)$ holomorphic at the origin. Here the equation is said to be singular if $a_{l}(0)=0$ $(j=1, \ldots, d)$. In this case, it is known that under the so-called Poincare condition, if $\left\{a_{l}(x)\right\}_{t=1}^{d}$ generates a simple ideal, every formal solution is convergent. However if we remove these conditions, we shall see that the formal solution, if it exists, may be divergent. More precisely, we will characterize the rate of divergence of formal solutions via Gevrey order of formal solutions determined by a Newton Polyhedron, a generalization of Newton Polygon which is familiar in the study of ordinary differential equations with an irregular singular point.
\end{abstract}

\section{§1. Introduction and Main Result}

In this paper we are concerned with a formal power series solution of the following equation

$$
P(x, D) u(x)=f(x),
$$

with $f$ holomorphic at the origin, where $P(x, D)$ is a first order linear partial differential operator with holomorphic coefficients at the origin:

Communicated by T. Kawai, June 28, 1999. Revised September 27, 1999.

1991 Mathematics Subject Classifications: primary 35C10, secondary 35A10.

* Graduate School of Mathematics, Nagoya University, Nagoya 464-8602, Japan. 


$$
P(x, D)=\sum_{i=1}^{d} a_{i}(x) D_{i}+b(x), \quad x=\left(x_{1}, \ldots, x_{d}\right) \in \mathbb{C}^{d}, \quad D_{i}=\frac{\partial}{\partial x_{i}}
$$

If $a_{i}(0) \neq 0$ for some $i$, the solvability is well known by Cauchy-Kowalevsky's Theorem. Therefore we shall study the case where $a_{i}(0)=0(\forall i)$, which is called a singular or degenerate case.

Concerning this problem, Oshima [8] studied a characterization of the kernel and cokernel of the mapping

$$
P(x, D): \mathcal{O} \rightarrow \mathcal{O},
$$

where $\mathcal{O}$ is the set of holomorphic functions at the origin. As main conditions, he assumed that $\left\{a_{i}(x)\right\}_{i=1}^{d}$ generates a proper and simple ideal of $\mathcal{O}$ and the so-called Poincaré condition for nonzero eigenvalues of the Jacobi matrix of coefficients at the origin. Various generalizations are made for higher order equations and nonlinear equations. The cases of higher order equations are studied by Miyake [4] and Miyake-Hashimoto [5]. In Gérard-Tahara [1] and Miyake-Shirai [6], the nonlinear equations are studied. Moreover, different characterizations of convergence of formal solutions of singular equations are obtained by Kashiwara-Kawai-Sjöstrand [3] and Miyake-Yoshino [7] for linear equations.

However, the cases without a Poincaré condition or a simple ideal condition have not been studied. We shall study these cases.

More precisely, let $D_{x} a(0):=\left(D_{i} a_{j}(0)\right)_{i, j=1, \ldots, d}$ be the Jacobi matrix at the origin of the mapping $a=\left(a_{1}, \ldots, a_{d}\right)$ and let its Jordan canonical form be

$$
\left(\begin{array}{lllll}
A & & & & \\
& B_{1} & & & \\
& & \ddots & & \\
& & & B_{k} & \\
& & & & O_{p}
\end{array}\right)
$$

where

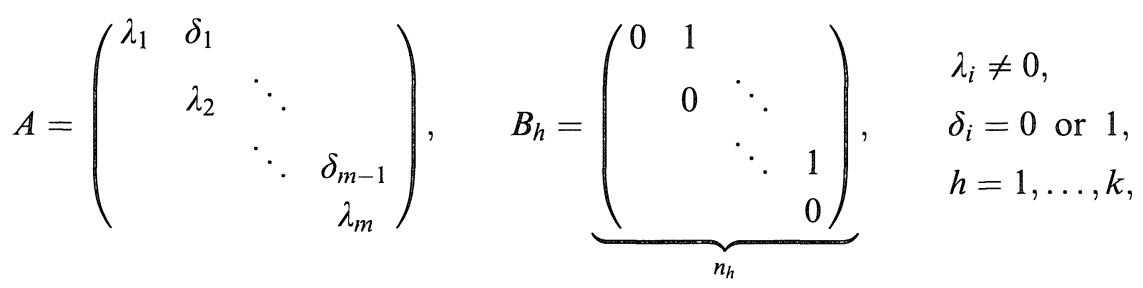

and $O_{p}$ is a zero-matrix of order $p\left(m, k, p \geq 0 ; n_{h} \geq 2 ; m+n_{1}+\cdots+n_{k}+p=d\right)$.

The Poincaré condition, in the above, demands

$$
0 \notin \operatorname{Ch}\left(\lambda_{1}, \ldots, \lambda_{m}\right),
$$


where $\operatorname{Ch}\left(\lambda_{1}, \ldots, \lambda_{m}\right)$ denotes the convex hull of the set $\left\{\lambda_{1}, \ldots, \lambda_{m}\right\}$ in the complex plane. We remark that this condition is equivalent to

$$
\left|\sum_{i=1}^{m} \lambda_{i} \alpha_{i}\right| \geq \delta|\alpha|, \quad \forall \alpha=\left(\alpha_{1}, \ldots, \alpha_{m}\right) \in \mathbf{N}^{m},
$$

for some positive constant $\delta$, where $\mathbf{N}=\{0,1,2, \ldots\}$.

In this paper we also assume (1.2) if $m \geq 1$. In order to ensure the unique existence of a formal power series solution of (1.1) we assume

$$
\sum_{i=1}^{m} \lambda_{i} \alpha_{i}+b(0) \neq 0, \quad \forall \alpha \in \mathbf{N}^{m}
$$

Note that $b(0) \neq 0$. Precisely, throughout this paper we assume the following condition (Po):

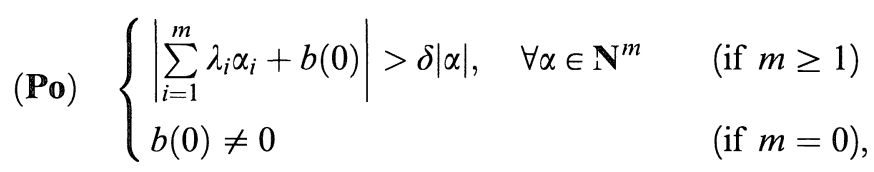

where $\delta$ is a positive constant independent of $\alpha \in \mathbf{N}^{m}$. It should be noted that under the condition (Po) the formal solution of (1.1) exists uniquely, while it is divergent in general as we will see in the following examples.

Examples. In the following examples, each independent variable denotes a one-dimensional complex variable.

(1) (the case $(m, k, p)=(0,0,2))$. For the equation

$$
\left(1-x^{2} D_{x}-y^{2} D_{y}\right) u(x, y)=x+y,
$$

we have the formal solution $u(x, y)=\sum_{m=1}^{\infty}(m-1) ! x^{m}+\sum_{n=1}^{\infty}(n-1) ! y^{n}$.

(2) (the case $(m, k, p)=(0,1,0))$. For

$$
\left(1-y D_{x}-x^{2} D_{x}\right) u(x, y)=x
$$

the formal solution $u(x, y)=\sum_{m, n \geq 0} u_{m n} x^{m} y^{n}$ satisfies $u(x, 0)=\sum_{m=1}^{\infty}(m-1) ! x^{m}$ and $u(0, y) \gg \sum_{n=1}^{\infty} n !(n-1) ! y^{n}$, where $A \gg B$ means that $A$ is a majorant series of $B$.

(3) (the case $(m, k, p)=(0,1,1))$. For

$$
\left(1-y D_{x}-x^{2} D_{x}-z^{2} D_{z}\right) u(x, y, z)=x+z,
$$

the formal solution $u(x, y, z)=\sum_{l, m, n \geq 0} u_{l m n} x^{l} y^{m} z^{n}$ satisfies $u(x, 0,0)=$ $\sum_{l=1}^{\infty}(l-1) ! x^{l}, u(0, y, 0) \gg \sum_{m=1}^{\infty} m !(m-1) ! y^{m}$ and $u(0,0, z)=\sum_{n=1}^{\infty}(n-1) ! z^{n}$.

(4) (the case $(m, k, p)=(1,0,1))$. For

$$
\left(1+x D_{x}-x^{2} D_{y}-y^{2} D_{y}\right) u(x, y)=y,
$$


the formal solution $u(x, y)=\sum_{m, n \geq 0} u_{m n} x^{m} y^{n}$ satisfies $u(x, 0) \gg \sum_{m=1}^{\infty} 2^{-m}$. $(m-1) ! x^{2 m}$ and $u(0, y)=\sum_{n=1}^{\infty}(n-1) ! y^{n}$.

(5) (the case $(m, k, p)=(1,1,0))$. For

$$
\left(1+x D_{x}-z D_{y}-x^{2} D_{y}-y^{2} D_{y}\right) u(x, y, z)=y,
$$

the formal solution $u(x, y, z)=\sum_{l, m, n \geq 0} u_{l m n} x^{l} y^{m} z^{n}$ satisfies $u(x, 0,0) \gg \sum_{l=1}^{\infty}$. $2^{-l}(l-1) ! x^{2 l}, u(0, y, 0)=\sum_{m=1}^{\infty}(m-1) ! y^{m}$ and $u(0,0, z) \gg \sum_{n=1}^{\infty} n !(n-1) ! z^{n}$.

(6) (the case $(m, k, p)=(1,1,1))$. For

$$
\left(1+x D_{x}-z D_{y}-x^{2} D_{y}-y^{2} D_{y}-w^{2} D_{w}\right) u(x, y, z, w)=y+w,
$$

the formal solution $u(x, y, z, w)=\sum_{k, l, m, n \geq 0} u_{k l m n} x^{k} y^{l} z^{m} w^{n}$ satisfies $u(x, 0,0,0)$ $\gg \sum_{k=1}^{\infty} 2^{-k}(k-1) ! x^{2 k}, \quad u(0, y, 0,0)=\sum_{l=1}^{\infty}(l-1) ! y^{l}, \quad u(0,0, z, 0) \gg \sum_{m=1}^{\infty} m !$. $(m-1) ! z^{m}$ and $u(0,0,0, w)=\sum_{n=1}^{\infty}(n-1) ! w^{n}$.

These results are proved by using the recursion formulas for the Taylor coefficients of formal solutions and by estimating carefully the coefficients from below.

We note that the Gevrey order gives the rate of divergence for a formal power series. We say that the formal power series $u(x)=\sum_{\alpha \in \mathbf{N}^{d}} u_{\alpha} x^{\alpha}\left(x^{\alpha}=\right.$ $\left.x_{1}^{\alpha_{1}} \ldots x_{d}^{\alpha_{d}}\right)$ belongs to $G^{(\mathbf{s})}\left(\mathbf{s}=\left(s_{1}, \ldots, s_{d}\right) \in \mathbf{R}^{d}\right)$, if the power series

$$
v(x)=\sum_{\alpha \in \mathbf{N}^{d}} u_{\alpha} \frac{x^{\alpha}}{(\alpha !)^{\mathbf{s}-1}}
$$

converges in a neighborhood of the origin, where $(\alpha !)^{\mathbf{s}-1}=\alpha_{1} !^{s_{1}-1} \ldots \alpha_{d} !^{s_{d}-1}$.

The main theorem in this paper is the following.

Theorem 1.1. Under the condition (Po), the equation (1.1) has a unique formal solution which belongs to $G^{(2 N, \ldots, 2 N)}$, where

$$
N= \begin{cases}\max \left\{n_{1}, \ldots, n_{k}\right\} & (\text { if } k \geq 1) \\ 1 & (\text { if } k=0 \text { and } p \geq 1) \\ 1 / 2 & (\text { if } k=p=0) .\end{cases}
$$

Remark 1. By our theorem we know that there exists a unique holomorphic solution if $k=p=0$, which is a special case of Oshima [8]. In fact, he studied the case $k=0$ in our notation under the assumption that $\left\{a_{i}(x)\right\}_{i=1}^{d}$ generates a proper and simple ideal of $\mathcal{O}$. Here we have to mention that if we remove the assumption that $\left\{a_{i}(x)\right\}_{i=1}^{d}$ generates a simple ideal, the formal solution does not necessarily converge, and it belongs to $G^{(2, \ldots, 2)}$ as shown in Example (4).

Remark 2. Precise estimates of Gevrey order in individual variables will be 
given in Theorem 2.1 after a linear transform of independent variables, which reduces $D_{x} a(0)$ to its Jordan canonical form.

Remark 3. Our main result is a generalization of Hibino [2], where the case $(m, k, p)=(0,1,0)$ (nilpotent matrix of maximal rank) is studied. In [2], we also study an asymptotic theory of formal solutions for a special type of equations, which will be published in the forthcoming paper.

\section{§2. Linear Transform of Operator and Newton Polyhedron}

In order to prove Theorem 1.1 we shall transform the operator $P(x, D)$ by a linear transform of variables which reduces $D_{x} a(0)$ to its Jordan canonical form. A reduced operator $P_{1}$ takes seven forms according to the values of $m, k$ and $p$, as follows:

(i) The case $m \geq 1, k \geq 1, p \geq 1$ :

$$
P_{1}=\sum_{i=1}^{m} \lambda_{i} x_{i} \frac{\partial}{\partial x_{i}}+b(0)+b_{1}+P_{1}^{\prime}+P_{1}^{\prime \prime}+P_{1}^{\prime \prime \prime}+P_{1}^{\prime \prime \prime \prime},
$$

where

$$
\begin{aligned}
& P_{1}^{\prime}=\sum_{i=1}^{m-1} \delta_{i} x_{i+1} \frac{\partial}{\partial x_{i}}+\sum_{i=1}^{m}\left(\sum_{|\alpha|+\left|\beta^{1}\right|+\cdots+\left|\beta^{k}\right|+|\gamma| \geq 2,|\alpha| \geq 1}^{\text {finite }} c_{i \alpha \beta^{1} \ldots \beta^{k} \gamma}\left(x, y^{1}, \ldots, y^{k}, z\right)\right. \\
& \left.\times x^{\alpha}\left(y^{1}\right)^{\beta^{1}} \ldots\left(y^{k}\right)^{\beta^{k}} z^{\gamma}\right) \frac{\partial}{\partial x_{i}} \\
& P_{1}^{\prime \prime}=\sum_{h=1}^{k} \sum_{j_{h}=1}^{n_{h}}\left(\sum_{|\alpha|+\left|\beta^{1}\right|+\cdots+\left|\beta^{k}\right|+|\gamma| \geq 2,|\alpha| \geq 1}^{\text {finite }} d_{j_{h} \alpha \beta^{1} \ldots \beta^{h} \gamma}\left(x, y^{1}, \ldots, y^{k}, z\right)\right. \\
& \left.\times x^{\alpha}\left(y^{1}\right)^{\beta^{1}} \ldots\left(y^{k}\right)^{\beta^{k}} z^{\gamma}\right) \frac{\partial}{\partial y_{j_{h}}^{h}} \\
& +\sum_{q=1}^{p}\left(\sum_{|\alpha|+\left|\beta^{1}\right|+\cdots+\left|\beta^{k}\right|+|\gamma| \geq 2,|\alpha| \geq 1}^{\text {finite }} e_{q \alpha \beta^{1} \ldots \beta^{k} \gamma}\left(x, y^{1}, \ldots, y^{k}, z\right)\right. \\
& \left.\times x^{\alpha}\left(y^{1}\right)^{\beta^{1}} \ldots\left(y^{k}\right)^{\beta^{k}} z^{\gamma}\right) \frac{\partial}{\partial z_{q}},
\end{aligned}
$$




$$
\begin{aligned}
& P_{1}^{\prime \prime \prime}=\sum_{h=1}^{k} \sum_{j_{h}=1}^{n_{h}-1} y_{j_{h}+1}^{h} \frac{\partial}{\partial y_{j_{h}}^{h}}+\sum_{h=1}^{k} \sum_{j_{h}=1}^{n_{h}}\left(\sum_{\left|\beta^{1}\right|+\cdots+\left|\beta^{k}\right|+|\gamma| \geq 2}^{\text {finite }} d_{j_{h} \beta^{1} \ldots \beta^{k}}\left(x, y^{1}, \ldots, y^{k}, z\right)\right. \\
& \left.\times\left(y^{1}\right)^{\beta^{1}} \ldots\left(y^{k}\right)^{\beta^{k}} z^{\gamma}\right) \frac{\partial}{\partial y_{j_{h}}^{h}} \\
& +\sum_{q=1}^{p}\left(\sum_{\left|\beta^{1}\right|+\cdots+\left|\beta^{k}\right|+|\gamma| \geq 2}^{\text {finite }} e_{q \beta^{1} \ldots \beta^{k} \gamma}\left(x, y^{1}, \ldots, y^{k}, z\right)\left(y^{1}\right)^{\beta^{1}} \ldots\left(y^{k}\right)^{\beta^{k}} z^{\gamma}\right) \frac{\partial}{\partial z_{q}}, \\
& P_{1}^{\prime \prime \prime \prime}=\sum_{i=1}^{m}\left(\sum_{\left|\beta^{1}\right|+\cdots+\left|\beta^{k^{\prime}}+\right| \gamma \mid \geq 2}^{\text {finite }} c_{i \beta^{1} \ldots \beta^{k} \gamma}\left(x, y^{1}, \ldots, y^{k}, z\right)\left(y^{1}\right)^{\beta^{1}} \ldots\left(y^{k}\right)^{\beta^{k}} z^{\gamma}\right) \frac{\partial}{\partial x_{i}}, \\
& b_{1}=b_{1}\left(x, y^{1}, \ldots, y^{k}, z\right) \\
& =\sum_{|\alpha|+\left|\beta^{1}\right|+\cdots+\left|\beta^{k}\right|+|\gamma| \geq 1}^{\text {finite }} b_{\alpha \beta^{1} \ldots \beta^{k}}\left(x, y^{1}, \ldots, y^{k}, z\right) x^{\alpha}\left(y^{1}\right)^{\beta^{1}} \ldots\left(y^{k}\right)^{\beta^{k}} z^{\gamma},
\end{aligned}
$$

where all coefficients $c_{i \alpha \beta^{1} \ldots \beta^{k} \gamma}$, etc., are holomorphic at the origin, and none of them vanish at the origin unless they vanish identically. In the following expressions, assume the same conditions for those functions appearing in the coefficients.

(ii) The case $m \geq 1, k \geq 1, p=0$ :

$$
P_{1}=\sum_{i=1}^{m} \lambda_{i} x_{i} \frac{\partial}{\partial x_{i}}+b(0)+b_{1}+P_{1}^{\prime}+P_{1}^{\prime \prime}+P_{1}^{\prime \prime \prime}+P_{1}^{\prime \prime \prime \prime},
$$

where

$$
\begin{aligned}
& P_{1}^{\prime}=\sum_{i=1}^{m-1} \delta_{i} x_{i+1} \frac{\partial}{\partial x_{i}}+\sum_{i=1}^{m}\left(\sum_{|\alpha|+\left|\beta^{1}\right|+\cdots+\left|\beta^{k}\right| \geq 2,|\alpha| \geq 1}^{\text {finite }} c_{i \alpha \beta^{1} \ldots \beta^{k}}\left(x, y^{1}, \ldots, y^{k}\right)\right. \\
& \left.\times x^{\alpha}\left(y^{1}\right)^{\beta^{1}} \ldots\left(y^{k}\right)^{\beta^{k}}\right) \frac{\partial}{\partial x_{i}} \\
& P_{1}^{\prime \prime}=\sum_{h=1}^{k} \sum_{j_{h}=1}^{n_{h}}\left(\sum_{|\alpha|+\left|\beta^{1}\right|+\cdots+\left|\beta^{k}\right| \geq 2,|\alpha| \geq 1}^{\text {finite }} d_{j_{h} \alpha \beta^{1} \ldots \beta^{k}}\left(x, y^{1}, \ldots, y^{k}\right)\right. \\
& \left.\times x^{\alpha}\left(y^{1}\right)^{\beta^{1}} \ldots\left(y^{k}\right)^{\beta^{k}}\right) \frac{\partial}{\partial y_{j_{h}}^{h}},
\end{aligned}
$$




$$
\begin{aligned}
P_{1}^{\prime \prime \prime}= & \sum_{h=1}^{k} \sum_{j_{h}=1}^{n_{h}-1} y_{j_{h}+1}^{h} \frac{\partial}{\partial y_{j_{h}}^{h}} \\
& +\sum_{h=1}^{k} \sum_{j_{h}=1}^{n_{h}}\left(\sum_{\left|\beta^{1}\right|+\ldots+\left|\beta^{k}\right| \geq 2}^{\text {finite }} d_{j_{h} \beta^{1} \ldots \beta^{k}}\left(x, y^{1}, \ldots, y^{k}\right)\left(y^{1}\right)^{\beta^{1}} \ldots\left(y^{k}\right)^{\beta^{k}}\right) \frac{\partial}{\partial y_{j_{h}}^{h}}, \\
P_{1}^{\prime \prime \prime \prime}= & \sum_{i=1}^{m}\left(\sum_{\left|\beta^{1}\right|+\cdots+\left|\beta^{k}\right| \geq 2}^{\text {finite }} c_{i \beta^{1} \ldots \beta^{k}}\left(x, y^{1}, \ldots, y^{k}\right)\left(y^{1}\right)^{\beta^{1}} \ldots\left(y^{k}\right)^{\beta^{k}}\right) \frac{\partial}{\partial x_{i}} \\
b_{1}= & b_{1}\left(x, y^{1}, \ldots, y^{k}\right) \\
= & \sum_{|\alpha|+\left|\beta^{1}\right|+\cdots+\left|\beta^{k}\right| \geq 1}^{\text {finite }} b_{\alpha \beta^{1} \ldots \beta^{k}}\left(x, y^{1}, \ldots, y^{k}\right) x^{\alpha}\left(y^{1}\right)^{\beta^{1}} \ldots\left(y^{k}\right)^{\beta^{k}} .
\end{aligned}
$$

(iii) The case $m \geq 1, k=0, p \geq 1$ :

$$
P_{1}=\sum_{i=1}^{m} \lambda_{i} x_{i} \frac{\partial}{\partial x_{i}}+b(0)+b_{1}+P_{1}^{\prime}+P_{1}^{\prime \prime}+P_{1}^{\prime \prime \prime}+P_{1}^{\prime \prime \prime \prime},
$$

where

$$
\begin{aligned}
P_{1}^{\prime} & =\sum_{i=1}^{m-1} \delta_{i} x_{i+1} \frac{\partial}{\partial x_{i}}+\sum_{i=1}^{m}\left(\sum_{|\alpha|+|\gamma| \geq 2,|\alpha| \geq 1}^{\text {finite }} c_{i \alpha \gamma}(x, z) x^{\alpha} z^{\gamma}\right) \frac{\partial}{\partial x_{i}} \\
P_{1}^{\prime \prime} & =\sum_{q=1}^{p}\left(\sum_{|\alpha|+|\gamma| \geq 2,|\alpha| \geq 1}^{\text {finite }} e_{q \alpha \gamma}(x, z) x^{\alpha} z^{\gamma}\right) \frac{\partial}{\partial z_{q}} \\
P_{1}^{\prime \prime \prime} & =\sum_{q=1}^{p}\left(\sum_{|\gamma| \geq 2}^{\text {finite }} e_{q \gamma}(x, z) z^{\gamma}\right) \frac{\partial}{\partial z_{q}} \\
P_{1}^{\prime \prime \prime \prime} & =\sum_{i=1}^{m}\left(\sum_{|\gamma| \geq 2}^{\text {finite }} c_{i \gamma}(x, z) z^{\gamma}\right) \frac{\partial}{\partial x_{i}} \\
b_{1} & =b_{1}(x, z)=\sum_{|\alpha|+|\gamma| \geq 1}^{\text {finite }} b_{\alpha \gamma}(x, z) x^{\alpha} z^{\gamma} .
\end{aligned}
$$

(iv) The case $m \geq 1, k=p=0$ :

$$
\begin{aligned}
P_{1}= & \sum_{i=1}^{m} \lambda_{i} x_{i} \frac{\partial}{\partial x_{i}}+b(0)+\sum_{|\alpha| \geq 1}^{\text {finite }} b_{\alpha}(x) x^{\alpha}+\sum_{i=1}^{m-1} \delta_{i} x_{i+1} \frac{\partial}{\partial x_{i}} \\
& +\sum_{i=1}^{m}\left(\sum_{|\alpha| \geq 2}^{\text {finite }} c_{i \alpha}(x) x^{\alpha}\right) \frac{\partial}{\partial x_{i}} .
\end{aligned}
$$


(v) The case $m=0, k \geq 1, p \geq 1$ :

$$
P_{1}=b(0)+b_{1}+P_{1}^{\prime \prime \prime},
$$

where

$$
\begin{aligned}
P_{1}^{\prime \prime \prime}= & \sum_{h=1}^{k} \sum_{j_{h}=1}^{n_{h}-1} y_{j_{h}+1}^{h} \frac{\partial}{\partial y_{j_{h}}^{h}}+\sum_{h=1}^{k} \sum_{j_{h}=1}^{n_{h}}\left(\sum_{\left|\beta^{1}\right|+\ldots+\left|\beta^{k}\right|+|\gamma| \geq 2}^{\text {finite }} d_{j_{h} \beta^{1} \ldots \beta^{k} \gamma}\left(y^{1}, \ldots, y^{k}, z\right)\right. \\
& \left.\times\left(y^{1}\right)^{\beta^{1}} \ldots\left(y^{k}\right)^{\beta^{k}} z^{\gamma}\right) \frac{\partial}{\partial y_{J_{h}^{h}}^{h}} \\
& +\sum_{q=1}^{p}\left(\sum_{\left|\beta^{1}\right|+\cdots+\left|\beta^{k}\right|+|\gamma| \geq 2}^{\text {finite }} e_{q \beta^{1} \ldots \beta^{k} \gamma}\left(y^{1}, \ldots, y^{k}, z\right)\left(y^{1}\right)^{\left.\beta^{1} \ldots\left(y^{k}\right)^{\beta^{k}} z^{\gamma}\right) \frac{\partial}{\partial z_{q}}},\right. \\
b_{1}= & b_{1}\left(y^{1}, \ldots, y^{k}, z\right) \\
= & \sum_{\left|\beta^{1}\right|+\cdots+\left|\beta^{k}\right|+|\gamma| \geq 1}^{\text {finite }} b_{\beta^{1} \ldots \beta^{k} \gamma}\left(y^{1}, \ldots, y^{k}, z\right)\left(y^{1}\right)^{\beta^{1} \ldots\left(y^{k}\right)^{\beta^{k}} z^{\gamma} .}
\end{aligned}
$$

(vi) The case $m=0, k \geq 1, p=0$ :

$$
P_{1}=b(0)+b_{1}+P_{1}^{\prime \prime \prime}
$$

where

$$
\begin{aligned}
P_{1}^{\prime \prime \prime}= & \sum_{h=1}^{k} \sum_{j_{h}=1}^{n_{h}-1} y_{j_{h}+1}^{h} \frac{\partial}{\partial y_{j_{h}}^{h}} \\
& +\sum_{h=1}^{k} \sum_{j_{h}=1}^{n_{h}}\left(\sum_{\left|\beta^{1}\right|+\ldots+\left|\beta^{k}\right| \geq 2}^{\text {finite }} d_{j_{h} \beta^{1} \ldots \beta^{k}}\left(y^{1}, \ldots, y^{k}\right)\left(y^{1}\right)^{\beta^{1}} \ldots\left(y^{k}\right)^{\beta^{k}}\right) \frac{\partial}{\partial y_{j_{h}}^{h}} \\
b_{1}= & b_{1}\left(y^{1}, \ldots, y^{k}\right) \\
= & \sum_{\left|\beta^{1}\right|+\cdots+\left|\beta^{k}\right| \geq 1}^{\text {finite }} b_{\beta^{1} \ldots \beta^{k}}\left(y^{1}, \ldots, y^{k}\right)\left(y^{1}\right)^{\beta^{1}} \ldots\left(y^{k}\right)^{\beta^{k}}
\end{aligned}
$$

(vii) The case $m=k=0, p \geq 1$ :

$$
P_{1}=b(0)+b_{1}+P_{1}^{\prime \prime \prime},
$$


where

$$
\begin{aligned}
P_{1}^{\prime \prime \prime} & =\sum_{q=1}^{p}\left(\sum_{|\gamma| \geq 2}^{\text {finite }} e_{q \gamma}(z) z^{\gamma}\right) \frac{\partial}{\partial z_{q}} \\
b_{1} & =b_{1}(z)=\sum_{|\gamma| \geq 1}^{\text {finite }} b_{\gamma}(z) z^{\gamma} .
\end{aligned}
$$

Now we shall study the equation

$$
P_{1} u=f
$$

with $f$ holomorphic at the origin.

In order to give the Gevrey order in an individual variable for formal solutions of (2.1), we study the Newton Polyhedron of the operator, which is a generalization of the Newton Polyhedron introduced by H. Yamazawa [9].

Newton Polyhedron. Let

$$
P\left(\xi, D_{\xi}\right)=\sum^{\text {finite }} a_{\alpha \beta}(\xi) \xi^{\alpha} D_{\xi}^{\beta}
$$

$\left(\xi=\left(\xi_{1}, \ldots, \xi_{d}\right), D_{\xi}^{\beta}=\left(\partial / \partial \xi_{1}\right)^{\beta_{1}} \ldots\left(\partial / \partial \xi_{d}\right)^{\beta_{d}}\right)$ be a linear partial differential operator, where all coefficients are holomorphic at the origin and do not vanish at the origin unless they vanish identically.

We put

$$
Q(\alpha, \beta)=\left\{(\mathscr{X}, \mathscr{Y})=\left(\mathscr{X}_{1}, \ldots, \mathscr{X}_{d}, \mathscr{Y}\right) \in \mathbf{R}^{d+1} ; \mathscr{X}_{i} \geq \alpha_{i}-\beta_{i}, \mathscr{Y} \leq|\beta|\right\}
$$

and define the Newton Polyhedron $N(P)$ by

$$
N(P)=\operatorname{Ch}\left\{Q(\alpha, \beta) ;(\alpha, \beta) \text { with } a_{\alpha \beta} \not \equiv 0\right\},
$$

where $\operatorname{Ch}(A)$ denotes the convex hull of a set $A \subset \mathbf{R}^{d+1}$.

Now we shall apply the above general definition to the equation (2.1) for the cases (i)-(vii). We remark that the correspondence of variables between $\left(x, y^{1}, \ldots, y^{k}, z\right)$ and $\xi$ is given by

\begin{tabular}{|l|c|}
\hline & $\xi$ \\
\hline (i) & $\left(x, y^{1}, \ldots, y^{k}, z\right)$ \\
(ii) & $\left(x, y^{1}, \ldots, y^{k}\right)$ \\
(iii) & $(x, z)$ \\
(iv) & - \\
(v) & $\left(y^{1}, \ldots, y^{k}, z\right)$ \\
(vi) & $\left(y^{1}, \ldots, y^{k}\right)$ \\
(vii) & $z$ \\
\hline
\end{tabular}


In order to state the main theorem in this section, we shall define the sets $S_{i}$ $(i=1,2,3,5,6$ and 7$), \tilde{S}_{j}, S_{j}^{\prime}$ and $S_{j}^{\prime \prime}(j=1,2$ and 3$)$ whose elements give the Gevrey order of formal solutions of (2.1).

(i) For $\left(\boldsymbol{\rho}, \boldsymbol{\sigma}^{1}, \ldots, \boldsymbol{\sigma}^{k}, \boldsymbol{\tau}\right) \in[1, \infty)^{d}\left(\boldsymbol{\rho}=\left(\rho_{1}, \ldots, \rho_{m}\right), \boldsymbol{\sigma}^{h}=\left(\sigma_{1}^{h}, \ldots, \sigma_{n_{h}}^{h}\right)\right.$ $\left.(h=1, \ldots, k), \tau=\left(\tau_{1}, \ldots, \tau_{p}\right)\right)$ we put

$$
\begin{aligned}
\tilde{\Lambda}_{1}\left(\boldsymbol{\rho}, \boldsymbol{\sigma}^{1}, \ldots, \boldsymbol{\sigma}^{k}, \boldsymbol{\tau}\right)= & \left\{\left(\mathscr{X}, \mathscr{Y}^{1}, \ldots, \mathscr{Y}^{k}, \mathscr{Z}, \mathscr{W}\right) \in \mathbb{R}^{d+1} ;(\boldsymbol{\rho}-\mathbb{1}) \cdot \mathscr{X}\right. \\
& \left.+\sum_{h=1}^{k}\left(\boldsymbol{\sigma}^{h}-\mathbb{1}\right) \cdot \mathscr{Y}^{h}+(\boldsymbol{\tau}-\mathbb{1}) \cdot \mathscr{Z}-\mathscr{W} \geq-1\right\}, \\
\Lambda_{1}\left(\boldsymbol{\rho}, \boldsymbol{\sigma}^{1}, \ldots, \boldsymbol{\sigma}^{k}, \boldsymbol{\tau}\right)= & \left\{\left(\mathscr{X}, \mathscr{Y}^{1}, \ldots, \mathscr{Y}^{k}, \mathscr{Z}, \mathscr{W}\right) \in \mathbb{R}^{d+1} ;(\boldsymbol{\rho}-\mathbb{1}) \cdot \mathscr{X}\right. \\
& \left.+\sum_{h=1}^{k}\left(\boldsymbol{\sigma}^{h}-\mathbb{1}\right) \cdot \mathscr{Y}^{h}+(\boldsymbol{\tau}-\mathbb{1}) \cdot \mathscr{Z}-\mathscr{W} \geq 0\right\},
\end{aligned}
$$

where $\mathbb{1}=(1, \ldots, 1)$ and define

$$
\begin{aligned}
\tilde{S}_{1} & =\left\{\left(\boldsymbol{\rho}, \boldsymbol{\sigma}^{1}, \ldots, \boldsymbol{\sigma}^{k}, \boldsymbol{\tau}\right) ; N\left(P_{1}^{\prime}+P_{1}^{\prime \prime}\right) \subset \tilde{\Lambda}_{1}\left(\boldsymbol{\rho}, \boldsymbol{\sigma}^{1}, \ldots, \boldsymbol{\sigma}^{k}, \boldsymbol{\tau}\right)\right\}, \\
S_{1} & =\left\{\left(\boldsymbol{\rho}, \boldsymbol{\sigma}^{1}, \ldots, \boldsymbol{\sigma}^{k}, \boldsymbol{\tau}\right) ; N\left(P_{1}^{\prime \prime \prime}\right) \subset \Lambda_{1}\left(\boldsymbol{\rho}, \boldsymbol{\sigma}^{1}, \ldots, \boldsymbol{\sigma}^{k}, \boldsymbol{\tau}\right)\right\}, \\
S_{1}^{\prime} & =\left\{\left(\boldsymbol{\rho}, \boldsymbol{\sigma}^{1}, \ldots, \boldsymbol{\sigma}^{k}, \boldsymbol{\tau}\right) ; N\left(P_{1}^{\prime \prime \prime}\right) \subset \tilde{\Lambda}_{1}\left(\boldsymbol{\rho}, \boldsymbol{\sigma}^{1}, \ldots, \boldsymbol{\sigma}^{k}, \boldsymbol{\tau}\right)\right\}, \\
S_{1}^{\prime \prime} & =\left\{\left(\boldsymbol{\rho}, \boldsymbol{\sigma}^{1}, \ldots, \boldsymbol{\sigma}^{k}, \boldsymbol{\tau}\right) ; N\left(P_{1}^{\prime \prime \prime}\right) \subset \Lambda_{1}\left(\boldsymbol{\rho}, \boldsymbol{\sigma}^{1}, \ldots, \boldsymbol{\sigma}^{k}, \boldsymbol{\tau}\right)\right\} .
\end{aligned}
$$

(ii) For $\left(\boldsymbol{\rho}, \boldsymbol{\sigma}^{1}, \ldots, \boldsymbol{\sigma}^{k}\right) \in[1, \infty)^{d}$ we put

$$
\begin{aligned}
\tilde{\Lambda}_{2}\left(\boldsymbol{\rho}, \boldsymbol{\sigma}^{1}, \ldots, \boldsymbol{\sigma}^{k}\right)= & \left\{\left(\mathscr{X}, \mathscr{Y}^{1}, \ldots, \mathscr{Y}^{k}, \mathscr{W}\right) \in \mathbb{R}^{d+1} ;(\boldsymbol{\rho}-\mathbb{1}) \cdot \mathscr{X}\right. \\
& \left.+\sum_{h=1}^{k}\left(\boldsymbol{\sigma}^{h}-\mathbb{1}\right) \cdot \mathscr{Y}^{h}-\mathscr{W} \geq-1\right\}, \\
\Lambda_{2}\left(\boldsymbol{\rho}, \boldsymbol{\sigma}^{1}, \ldots, \boldsymbol{\sigma}^{k}\right)= & \left\{\left(\mathscr{X}, \mathscr{Y}^{1}, \ldots, \mathscr{Y}^{k}, \mathscr{W}\right) \in \mathbb{R}^{d+1} ;(\boldsymbol{\rho}-\mathbb{1}) \cdot \mathscr{X}\right. \\
& \left.+\sum_{h=1}^{k}\left(\boldsymbol{\sigma}^{h}-\mathbb{1}\right) \cdot \mathscr{Y}^{h}-\mathscr{W} \geq 0\right\}
\end{aligned}
$$


and define

$$
\begin{aligned}
& \tilde{S}_{2}=\left\{\left(\boldsymbol{\rho}, \boldsymbol{\sigma}^{1}, \ldots, \boldsymbol{\sigma}^{k}\right) ; N\left(P_{1}^{\prime}+P_{1}^{\prime \prime}\right) \subset \tilde{\Lambda}_{2}\left(\boldsymbol{\rho}, \boldsymbol{\sigma}^{1}, \ldots, \boldsymbol{\sigma}^{k}\right)\right\} \\
& S_{2}=\left\{\left(\boldsymbol{\rho}, \boldsymbol{\sigma}^{1}, \ldots, \boldsymbol{\sigma}^{k}\right) ; N\left(P_{1}^{\prime \prime \prime}\right) \subset \Lambda_{2}\left(\boldsymbol{\rho}, \boldsymbol{\sigma}^{1}, \ldots, \boldsymbol{\sigma}^{k}\right)\right\} \\
& S_{2}^{\prime}=\left\{\left(\boldsymbol{\rho}, \boldsymbol{\sigma}^{1}, \ldots, \boldsymbol{\sigma}^{k}\right) ; N\left(P_{1}^{\prime \prime \prime \prime}\right) \subset \tilde{\Lambda}_{2}\left(\boldsymbol{\rho}, \boldsymbol{\sigma}^{1}, \ldots, \boldsymbol{\sigma}^{k}\right)\right\} \\
& S_{2}^{\prime \prime}=\left\{\left(\boldsymbol{\rho}, \boldsymbol{\sigma}^{1}, \ldots, \boldsymbol{\sigma}^{k}\right) ; N\left(P_{1}^{\prime \prime \prime \prime}\right) \subset \Lambda_{2}\left(\boldsymbol{\rho}, \boldsymbol{\sigma}^{1}, \ldots, \boldsymbol{\sigma}^{k}\right)\right\} .
\end{aligned}
$$

(iii) For $(\boldsymbol{\rho}, \boldsymbol{\tau}) \in[1, \infty)^{d}$ put

$$
\begin{aligned}
& \tilde{\Lambda}_{3}(\boldsymbol{\rho}, \boldsymbol{\tau})=\left\{(\mathscr{X}, \mathscr{Z}, \mathscr{W}) \in \mathbf{R}^{d+1} ;(\boldsymbol{\rho}-\mathbf{1}) \cdot \mathscr{X}+(\boldsymbol{\tau}-\mathbf{1}) \cdot \mathscr{Z}-\mathscr{W} \geq-1\right\}, \\
& \Lambda_{3}(\boldsymbol{\rho}, \boldsymbol{\tau})=\left\{(\mathscr{X}, \mathscr{Z}, \mathscr{W}) \in \mathbf{R}^{d+1} ;(\boldsymbol{\rho}-\mathbf{1}) \cdot \mathscr{X}+(\boldsymbol{\tau}-\mathbf{1}) \cdot \mathscr{Z}-\mathscr{W} \geq 0\right\}
\end{aligned}
$$

and define

$$
\begin{aligned}
& \tilde{S}_{3}=\left\{(\boldsymbol{\rho}, \boldsymbol{\tau}) ; N\left(P_{1}^{\prime}+P_{1}^{\prime \prime}\right) \subset \tilde{\Lambda}_{3}(\boldsymbol{\rho}, \boldsymbol{\tau})\right\} \\
& S_{3}=\left\{(\boldsymbol{\rho}, \boldsymbol{\tau}) ; N\left(P_{1}^{\prime \prime \prime}\right) \subset \Lambda_{3}(\boldsymbol{\rho}, \boldsymbol{\tau})\right\} \\
& S_{3}^{\prime}=\left\{(\boldsymbol{\rho}, \boldsymbol{\tau}) ; N\left(P_{1}^{\prime \prime \prime \prime}\right) \subset \tilde{\Lambda}_{3}(\boldsymbol{\rho}, \boldsymbol{\tau})\right\} \\
& S_{3}^{\prime \prime}=\left\{(\boldsymbol{\rho}, \boldsymbol{\tau}) ; N\left(P_{1}^{\prime \prime \prime \prime}\right) \subset \Lambda_{3}(\boldsymbol{\rho}, \boldsymbol{\tau})\right\} .
\end{aligned}
$$

(v) For $\left(\boldsymbol{\sigma}^{1}, \ldots, \boldsymbol{\sigma}^{k}, \boldsymbol{\tau}\right) \in[1, \infty)^{d}$ put

$$
\begin{aligned}
\Lambda_{5}\left(\boldsymbol{\sigma}^{1}, \ldots, \boldsymbol{\sigma}^{k}, \boldsymbol{\tau}\right)= & \left\{\left(\mathscr{Y}^{1}, \ldots, \mathscr{Y}^{k}, \mathscr{Z}, \mathscr{W}\right) \in \mathbf{R}^{d+1} ; \sum_{h=1}^{k}\left(\boldsymbol{\sigma}^{h}-\mathbf{1}\right) \cdot \mathscr{Y}^{h}\right. \\
& +(\boldsymbol{\tau}-\mathbb{1}) \cdot \mathscr{Z}-\mathscr{W} \geq 0\}
\end{aligned}
$$

and define

$$
S_{5}=\left\{\left(\boldsymbol{\sigma}^{1}, \ldots, \boldsymbol{\sigma}^{k}, \boldsymbol{\tau}\right) ; N\left(P_{1}^{\prime \prime \prime}\right) \subset \Lambda_{5}\left(\boldsymbol{\sigma}^{1}, \ldots, \boldsymbol{\sigma}^{k}, \boldsymbol{\tau}\right)\right\} .
$$

(vi) For $\left(\sigma^{1}, \ldots, \sigma^{k}\right) \in[1, \infty)^{d}$ we put

$$
\Lambda_{6}\left(\boldsymbol{\sigma}^{1}, \ldots, \boldsymbol{\sigma}^{k}\right)=\left\{\left(\mathscr{Y}^{1}, \ldots, \mathscr{Y}^{k}, \mathscr{W}\right) \in \mathbf{R}^{d+1} ; \sum_{h=1}^{k}\left(\boldsymbol{\sigma}^{h}-\mathbf{1}\right) \cdot \mathscr{Y}^{h}-\mathscr{W} \geq 0\right\}
$$

and define

$$
S_{6}=\left\{\left(\boldsymbol{\sigma}^{1}, \ldots, \boldsymbol{\sigma}^{k}\right) ; N\left(P_{1}^{\prime \prime \prime}\right) \subset \Lambda_{6}\left(\boldsymbol{\sigma}^{1}, \ldots, \boldsymbol{\sigma}^{k}\right)\right\} .
$$

(vii) For $\tau \in[1, \infty)^{d}$ put

$$
\Lambda_{7}(\boldsymbol{\tau})=\left\{(\mathscr{Z}, \mathscr{W}) \in \mathbf{R}^{d+1} ;(\boldsymbol{\tau}-\mathbf{1}) \cdot \mathscr{Z}-\mathscr{W} \geq 0\right\}
$$


and define

$$
S_{7}=\left\{\tau ; N\left(P_{1}^{\prime \prime \prime}\right) \subset \Lambda_{7}(\tau)\right\} .
$$

Then we obtain the following result:

Theorem 2.1. For each case (i)-(vii) let us assume the following condition:

(i ) $P_{1}^{\prime \prime \prime \prime}=0 \Rightarrow \mathbf{s}=\left(\boldsymbol{\rho}, \sigma^{1}, \ldots, \sigma^{k}, \tau\right) \in \tilde{S}_{1} \cap S_{1}$,

$$
\begin{aligned}
& P_{1}^{\prime \prime}=0 \Rightarrow \mathbf{s}=\left(\boldsymbol{\rho}, \boldsymbol{\sigma}^{1}, \ldots, \boldsymbol{\sigma}^{k}, \boldsymbol{\tau}\right) \in \tilde{S}_{1} \cap S_{1} \cap S_{1}^{\prime}, \\
& P_{1}^{\prime \prime \prime \prime}, P_{1}^{\prime \prime} \neq 0 \Rightarrow \mathbf{s}=\left(\boldsymbol{\rho}, \boldsymbol{\sigma}^{1}, \ldots, \boldsymbol{\sigma}^{k}, \boldsymbol{\tau}\right) \in \tilde{S}_{1} \cap S_{1} \cap S_{1}^{\prime \prime},
\end{aligned}
$$

(ii) $P_{1}^{\prime \prime \prime \prime}=0 \Rightarrow \mathbf{s}=\left(\boldsymbol{\rho}, \boldsymbol{\sigma}^{1}, \ldots, \boldsymbol{\sigma}^{k}\right) \in \tilde{S}_{2} \cap S_{2}$,

$P_{1}^{\prime \prime}=0 \Rightarrow \mathbf{s}=\left(\boldsymbol{\rho}, \boldsymbol{\sigma}^{1}, \ldots, \boldsymbol{\sigma}^{k}\right) \in \tilde{S}_{2} \cap S_{2} \cap S_{2}^{\prime}$,

$P_{1}^{\prime \prime \prime \prime}, P_{1}^{\prime \prime} \neq 0 \Rightarrow \mathbf{s}=\left(\boldsymbol{\rho}, \boldsymbol{\sigma}^{1}, \ldots, \boldsymbol{\sigma}^{k}\right) \in \tilde{S}_{2} \cap S_{2} \cap S_{2}^{\prime \prime}$,

(iii) $P_{1}^{\prime \prime \prime \prime}=0 \Rightarrow \mathbf{s}=(\boldsymbol{p}, \tau) \in \tilde{S}_{3} \cap S_{3}$,

$P_{1}^{\prime \prime}=0 \Rightarrow \mathbf{s}=(\boldsymbol{\rho}, \tau) \in \tilde{S}_{3} \cap S_{3} \cap S_{3}^{\prime}$,

$P_{1}^{\prime \prime \prime \prime}, P_{1}^{\prime \prime} \neq 0 \Rightarrow \mathbf{s}=(\boldsymbol{\rho}, \boldsymbol{\tau}) \in \tilde{S}_{3} \cap S_{3} \cap S_{3}^{\prime \prime}$,

(iv) $\mathbf{s}=(1, \ldots, 1)$,

(v) $\mathbf{s}=\left(\boldsymbol{\sigma}^{1}, \ldots, \boldsymbol{\sigma}^{k}, \boldsymbol{\tau}\right) \in S_{5}$,

(vi) $\mathbf{s}=\left(\boldsymbol{\sigma}^{1}, \ldots, \boldsymbol{\sigma}^{k}\right) \in S_{6}$,

(vii) $\mathbf{s}=\boldsymbol{\tau} \in S_{7}$.

Then the equation (2.1) with $f$ in $G^{(\mathbf{s})}$ has a unique formal solution which belongs to $G^{(\mathbf{s})}$.

Remark 1. In the case (iii), when $P_{1}^{\prime \prime \prime \prime}, P_{1}^{\prime \prime} \neq 0, \mathbf{s}=(\boldsymbol{p}, \tau)$ must belong to $\tilde{S}_{3} \cap S_{3} \cap S_{3}^{\prime \prime}$ not to $\tilde{S}_{3} \cap S_{3} \cap S_{3}^{\prime}$. For example, let us consider the equations

$$
\begin{array}{r}
\left(1+x D_{x}-y^{2} D_{x}\right) u(x, y)=f(x, y), \\
\left(1+x D_{x}-x^{2} D_{y}\right) u(x, y)=f(x, y), \\
\left(1+x D_{x}-x^{2} D_{y}-y^{2} D_{x}\right) u(x, y)=f(x, y),
\end{array}
$$

where $f(x, y)=\sum_{m, n \geq 0} x^{m} y^{n}(=(1 /(1-x))(1 /(1-y)))$. Here $-x^{2} D_{y}$ and $-y^{2} D_{x}$ correspond to $P_{1}^{\prime \prime}$ and $P_{1}^{\prime \prime \prime \prime}$, respectively. For (2.2) and (2.3), the formal solution converges, but for (2.4) it diverges. More precisely, the formal solution $u(x, y)=\sum_{m, n \geq 0} u_{m n} x^{m} y^{n}$ satisfies $u(0, y) \gg \sum_{n \geq 1} n ! y^{3 n}$, which is proved as follows: Let $u_{p}(x, y)=\sum_{m+n=p} u_{m, n}^{(p)} x^{m} y^{n}$ be the homogeneous part of $u(x, y)$. Then we obtain $u_{0}(x, y)=1, u_{1}(x, y)=(1 / 2) x+y$ and the following recursion formulas for $p \geq 2$ :

$$
\begin{aligned}
u_{p, 0}^{(p)} & =\frac{1}{1+p}\left(u_{p-2,1}^{(p-1)}+1\right), \\
u_{p-1,1}^{(p)} & =\frac{1}{p}\left(2 u_{p-3,2}^{(p-1)}+1\right),
\end{aligned}
$$




$$
\begin{aligned}
u_{p-j, j}^{(p)}= & \frac{1}{p-j+1}\left\{(j+1) u_{p-j-2, j+1}^{(p-1)}+(p-j+1) u_{p-j+1, j-2}^{(p-1)}+1\right\} \\
& j=2, \ldots, p-2, \\
u_{1, p-1}^{(p)}= & \frac{1}{2}\left(2 u_{2, p-3}^{(p-1)}+1\right), \\
u_{0, p}^{(p)}= & u_{1, p-2}^{(p-1)}+1 .
\end{aligned}
$$

Therefore it follows that

$$
\begin{aligned}
u_{0,3 n}^{(3 n)} & \geq u_{1,3 n-2}^{(3 n-1)} \geq u_{2,3 n-4}^{(3 n-2)} \geq \frac{3 n-3}{3} u_{0,3 n-3}^{(3 n-3)} \\
& \geq \frac{3 n-3}{3} \cdot \frac{3 n-6}{3} \cdots \frac{3 n-3 k}{3} u_{0,3 n-3 k}^{(3 n-3 k)} \\
& \geq \frac{3 n-3}{3} \cdots \frac{3 n-(3 n-3)}{3} u_{0,3}^{(3)} \\
& \geq(n-1) !
\end{aligned}
$$

which implies the conclusion.

The same is true for the cases (i) and (ii).

Remark 2. In each of the seven forms of the reduced operator $P_{1}$, if we assume that all coefficients of quadratic polynomials in the operators do not vanish, we get the biggest Gevrey order given by $\boldsymbol{\rho}_{0}=\overbrace{(N+1 / 2, \ldots, N+1 / 2)}^{m}$, $\boldsymbol{\rho}_{1}=\overbrace{(3 / 2, \ldots, 3 / 2)}^{m}, \sigma_{0}^{h}=\left(N+1, \ldots, N+n_{h}\right) \quad(h=1, \ldots, k), \tau_{0}=\overbrace{(N+1, \ldots, N+1)}^{p}$ and $\tau_{1}=\overbrace{(2, \ldots, 2)}$. The "biggest" means that if $\mathbf{s}$ satisfies the conditions in Theorem 2.1, the following inclusion holds for each case:

(i) $G^{(\mathbf{s})} \subset G^{\left(\boldsymbol{\rho}_{0}, \sigma_{0}^{1}, \ldots, \sigma_{0}^{k}, \tau_{0}\right)}$ (if $\left.P_{1}^{\prime \prime} \neq 0\right), G^{(\mathbf{s})} \subset G^{\left(\mathbf{1}, \sigma_{0}^{1}, \ldots, \sigma_{0}^{k}, \tau_{0}\right)}$ (if $P_{1}^{\prime \prime}=0$ ),

(ii) $G^{(\mathbf{s})} \subset G^{\left(\rho_{0}, \sigma_{0}^{1}, \ldots, \sigma_{0}^{k}\right)}$ (if $\left.P_{1}^{\prime \prime} \neq 0\right), G^{(\mathbf{s})} \subset G^{\left(\mathbf{1}, \sigma_{0}^{1}, \ldots, \sigma_{0}^{k}\right)}$ (if $P_{1}^{\prime \prime}=0$ ),

(iii) $G^{(\mathbf{s})} \subset G^{\left(\boldsymbol{\rho}_{1}, \tau_{1}\right)}$, (if $P_{1}^{\prime \prime} \neq 0$ ), $G^{(\mathbf{s})} \subset G^{\left(\mathbf{1}, \tau_{1}\right)}$, (if $P_{1}^{\prime \prime}=0$ ),

(iv) $G^{(\mathbf{s})}=G^{(\mathbf{1})}=\mathcal{O}$,

(v) $G^{(\mathbf{s})} \subset G^{\left(\sigma_{0}^{1}, \ldots, \sigma_{0}^{k}, \tau_{0}\right)}$,

(vi) $G^{(\mathbf{s})} \subset G^{\left(\sigma_{0}^{1}, \ldots, \sigma_{0}^{k}\right)}$,

(vii) $G^{(\mathbf{s})} \subset G^{\left(\tau_{1}\right)}$.

In the following examples we will determine Gevrey order concretely.

Let $\rho, \sigma_{1}, \sigma_{2}$ and $\tau$ denote the Gevrey order of the variables $x, y, z$ and $w$, respectively.

(1) (the case (vii))

$$
P_{1}=1+w^{2} D_{w}
$$


For the operator $w^{2} D_{w}$, we demand $(1-\tau)+(1-\tau)+\tau \leq 0$, that is, $\tau \geq 2$. Therefore we have $G^{(\tau)} \subset G^{(2)}$.

(2) (the case (vi))

$$
P_{1}=1+z D_{y}+y^{2} D_{z} .
$$

For the operators $z D_{y}$ and $y^{2} D_{z}$, we demand $\left(1-\sigma_{2}\right)+\sigma_{1} \leq 0$ and $\left(1-\sigma_{1}\right)+$ $\left(1-\sigma_{1}\right)+\sigma_{2} \leq 0$, respectively. Thus we obtain $G^{\left(\sigma_{1}, \sigma_{2}\right)} \subset G^{(3,4)}$.

(3) (the case (v))

$$
P_{1}=1+z D_{y}+y^{2} D_{z}+w^{2} D_{z} .
$$

For the operators $z D_{y}$ and $y^{2} D_{z}$, we demand the same inequalities as above, respectively. For $w^{2} D_{z}$, we assume $(1-\tau)+(1-\tau)+\sigma_{2} \leq 0$. It follows that $G^{\left(\sigma_{1}, \sigma_{2}, \tau\right)} \subset G^{(3,4,3)}$.

(4) (the case (iii), $P_{1}^{\prime \prime \prime \prime}=0$ )

$$
P_{1}=1+x D_{x}+x^{2} D_{w}+w^{2} D_{w} .
$$

For the operators $x D_{x}$ and $x^{2} D_{w}$, we demand $(1-\rho)+\rho \leq 1$ and $(1-\rho)+$ $(1-\rho)+\tau \leq 1$, respectively. For $w^{2} D_{w}$, we require $\tau \geq 2$ as in (1). These inequalities imply that $G^{(\rho, \tau)} \subset G^{(3 / 2,2)}$.

(5) (the case (iii), $P_{1}^{\prime \prime}=0$ )

$$
P_{1}=1+x D_{x}+w^{2} D_{w}+w^{2} D_{x} .
$$

For $x D_{x}$ and $w^{2} D_{w}$, we demand the same inequalities as above, while, for $w^{2} D_{x}$, we assume the inequality $(1-\tau)+(1-\tau)+\rho \leq 1$. Thus $G^{(\rho, \tau)} \subset G^{(1,2)}$ holds.

(6) (the case (iii), $P_{1}^{\prime \prime}, P_{1}^{\prime \prime \prime \prime} \neq 0$ )

$$
P_{1}=1+x D_{x}+x^{2} D_{w}+w^{2} D_{w}+w^{2} D_{x} .
$$

For $x D_{x}, x^{2} D_{w}$ and $x^{2} D_{w}$, the same inequalities are demanded, while, for $w^{2} D_{x}$, the inequality $(1-\tau)+(1-\tau)+\rho \leq 0$ is required instead of $(1-\tau)+(1-\tau)+$ $\rho \leq 1$. These inequalities imply that $G^{(\rho, \tau)} \subset G^{(3 / 2,2)}$.

(7) (the case (ii), $P_{1}^{\prime \prime \prime \prime}=0$ )

$$
P_{1}=1+x D_{x}+x^{2} D_{z}+z D_{y}+y^{2} D_{z} .
$$

For the operators $x D_{x}, x^{2} D_{z}, z D_{y}$ and $y^{2} D_{z}$, we demand $(1-\rho)+\rho \leq 1$, $(1-\rho)+(1-\rho)+\sigma_{2} \leq 1,\left(1-\sigma_{2}\right)+\sigma_{1} \leq 0$ and $\left(1-\sigma_{1}\right)+\left(1-\sigma_{1}\right)+\sigma_{2} \leq 0$, respectively. Thus we have $G^{\left(\rho, \sigma_{1}, \sigma_{2}\right)} \subset G^{(5 / 2,3,4)}$.

(8) (the case (ii), $P_{1}^{\prime \prime}=0$ )

$$
P_{1}=1+x D_{x}+z D_{y}+y^{2} D_{z}+z^{2} D_{x} .
$$

For $z^{2} D_{x}$, the inequality $\left(1-\sigma_{2}\right)+\left(1-\sigma_{2}\right)+\rho \leq 1$ is demanded. The other inequalities are the same as in (7). It follows that $G^{\left(\rho, \sigma_{1}, \sigma_{2}\right)} \subset G^{(1,3,4)}$. 
(9) (the case (ii), $P_{1}^{\prime \prime}, P_{1}^{\prime \prime \prime \prime} \neq 0$ )

$$
P_{1}=1+x D_{x}+x^{2} D_{z}+z D_{y}+y^{2} D_{z}+z^{2} D_{x} .
$$

For $z^{2} D_{x}$, we demand $\left(1-\sigma_{2}\right)+\left(1-\sigma_{2}\right)+\rho \leq 0$ instead of the inequality $\left(1-\sigma_{2}\right)+\left(1-\sigma_{2}\right)+\rho \leq 1$. By considering the same inequalities as in (7) for the other operators, we obtain $G^{\left(\rho, \sigma_{1}, \sigma_{2}\right)} \subset G^{(5 / 2,3,4)}$.

(10) (the case (i), $P_{1}^{\prime \prime \prime \prime}=0$ )

$$
P_{1}=1+x D_{x}+x^{2} D_{z}+z D_{y}+y^{2} D_{z}+w^{2} D_{z} .
$$

By the inequality $(1-\tau)+(1-\tau)+\sigma_{2} \leq 0$ demanded by $w^{2} D_{z}$ and those in (7), we have $G^{\left(\rho, \sigma_{1}, \sigma_{2}, \tau\right)} \subset G^{(5 / 2,3,4,3)}$.

(11) (the case (i), $P_{1}^{\prime \prime}=0$ )

$$
P_{1}=1+x D_{x}+z D_{y}+y^{2} D_{z}+w^{2} D_{z}+y^{2} D_{x} .
$$

By the inequality $\left(1-\sigma_{1}\right)+\left(1-\sigma_{1}\right)+\rho \leq 1$ demanded by $y^{2} D_{x}$ and those similar in (10), we obtain $G^{\left(\rho, \sigma_{1}, \sigma_{2}, \tau\right)} \subset G^{(1,3,4,3)}$.

(12) (the case (i), $P_{1}^{\prime \prime}, P_{1}^{\prime \prime \prime \prime} \neq 0$ )

$$
P_{1}=1+x D_{x}+x^{2} D_{z}+z D_{y}+y^{2} D_{z}+w^{2} D_{z}+y^{2} D_{x} .
$$

As for $y^{2} D_{x}$, we demand the inequality $\left(1-\sigma_{1}\right)+\left(1-\sigma_{1}\right)+\rho \leq 0$ instead of the one $\left(1-\sigma_{1}\right)+\left(1-\sigma_{1}\right)+\rho \leq 1$. For other operators, the same inequalities as in (10) are demanded. Thus $G^{\left(\rho, \sigma_{1}, \sigma_{2}, \tau\right)} \subset G^{(5 / 2,3,4,3)}$ holds.

By using the above inclusion relations in Gevrey spaces and by the change of independent variables we obtain Theorem 1.1 from Theorem 2.1 and the next Lemma 2.1. Thus the proof of Theorem 1.1 is reduced to that of Theorem 2.1.

Lemma 2.1. Let $u(x)=\sum_{\alpha \in \mathbf{N}^{d}} u_{\alpha} x^{\alpha} \in G^{(s, s, \ldots, s)} \quad(s \geq 1)$. Then for any linear transform $L=\left(l_{i j}\right)_{i, j=1, \ldots, d}: \mathbf{C}^{d} \rightarrow \mathbf{C}^{d}$, it holds that $v(y)=u(L y) \in$ $G^{(s, s, \ldots, s)}$.

Proof. First, we remark that $u(x) \in G^{(s, \ldots, s)}$ if and only if there exist some constants $C$ and $K$ such that

$$
\left|u^{(\alpha)}(0)\right| \leq C K^{|\alpha|}|\alpha| !^{s}, \quad \forall \alpha \in \mathbf{N}^{d},
$$

where $u^{(\alpha)}(x)=D_{x}^{\alpha} u(x)=\left(\partial / \partial x_{1}\right)^{\alpha_{1}} \ldots\left(\partial / \partial x_{d}\right)^{\alpha_{d}} u(x)$. By an easy calculation, we can obtain

$$
\left|v^{(\alpha)}(0)\right| \leq \mathscr{L} d^{|\alpha|}\left|u^{(\alpha)}(0)\right|,
$$

where $\mathscr{L}=\max \left\{l_{i j} ; i, j=1, \ldots, d\right\}$, which implies

$$
\left|v^{(\alpha)}(0)\right| \leq C \mathscr{L}(d K)^{|\alpha|}|\alpha| !^{s} .
$$

Therefore we obtain the Lemma. 
In order to prove Theorem 2.1 in the cases (i)-(vii), we will prove the cases (i), (ii), (iii) and (iv) by a same method. On the other hand, the cases (v), (vi) and (vii) are proved by the same method different from the one used for the cases (i)-(iv). Therefore we shall prove only the cases (ii) and (vi) in the following.

\section{§3. The Banach Spaces $G^{(\mathrm{s})}(\mathbb{R})$ and $\tilde{G}^{\left(\mathrm{s}^{1}, \mathrm{~s}^{2}\right)}\left(\mathbb{R}^{1}, \mathbb{R}^{2}\right)$}

Theorem 2.1 is proved by a contraction mapping principle in a Banach space. For this purpose we define two Banach spaces necessary in the proof, and we prove lemmas needed later.

Definition 3.1. Let $\mathbf{s}=\left(s_{1}, \ldots, s_{d}\right) \in \mathbf{R}_{+}^{d}\left(\mathbb{R}_{+}=\{r \in \mathbb{R} ; r \geq 0\}\right), \quad\left(\mathbf{s}^{1}, \mathbf{s}^{2}\right)=$ $\left(s_{1}^{1}, \ldots, s_{d_{1}}^{1}, s_{1}^{2}, \ldots, s_{d_{2}}^{2}\right) \in \mathbb{R}_{+}^{d_{1}+d_{2}}, \quad R=\left(R_{1}, \ldots, R_{d}\right) \in\left(\mathbb{R}_{+} \backslash\{0\}\right)^{d}$ and $\left(R^{1}, R^{2}\right)=$ $\left(R_{1}^{1}, \ldots, R_{d_{1}}^{1}, R_{1}^{2}, \ldots, R_{d_{2}}^{2}\right) \in\left(\mathbf{R}_{+} \backslash\{0\}\right)^{d_{1}+d_{2}}$. The spaces of formal power series $G^{(\mathbf{s})}(R)$ and $\tilde{G}^{\left(\mathbf{s}^{1}, \mathbf{s}^{2}\right)}\left(R^{1}, R^{2}\right)$ are defined as follows:

We say that $u(x)=\sum_{\alpha \in \mathbf{N}^{d}} u_{\alpha} x^{\alpha}$ belongs to $G^{(\mathbf{s})}(R)$ if

$$
\|u\|_{R}^{(\mathbf{s})} \equiv \sum_{\alpha \in \mathbf{N}^{d}}\left|u_{\alpha}\right| \frac{|\alpha| !}{(\mathbf{s} \cdot \alpha) !} R^{\alpha}<\infty
$$

$\left(|\alpha|=\alpha_{1}+\cdots+\alpha_{d}, \mathbf{s} \cdot \alpha=\sum_{i=1}^{d} s_{i} \alpha_{i}\right)$.

We say that $u(x, y)=\sum_{(\alpha, \beta) \in \mathbf{N}^{d_{1}+d_{2}}} u_{\alpha \beta} x^{\alpha} y^{\beta} \in \tilde{G}^{\left(\mathbf{s}^{1}, \mathbf{s}^{2}\right)}\left(R^{1}, R^{2}\right)$ if

$$
\left|\left\|u\left|\|_{R^{1}, R^{2}}^{\left(\mathbf{s}^{1}, \mathbf{s}^{2}\right)} \equiv \sum_{(\alpha, \beta) \in \mathbf{N}^{d_{1}+d_{2}}}\right| u_{\alpha \beta} \mid \frac{|\alpha| !|\beta| !}{\left(\mathbf{s}^{1} \cdot \alpha+\mathbf{s}^{2} \cdot \beta\right) !}\left(R^{1}\right)^{\alpha}\left(R^{2}\right)^{\beta}<\infty\right.\right.
$$

$\left(|\alpha|=\alpha_{1}+\cdots+\alpha_{d_{1}}, \quad|\beta|=\beta_{1}+\cdots+\beta_{d_{2}}, \mathbf{s}^{1} \cdot \alpha=\sum_{i=1}^{d_{1}} s_{i}^{1} \alpha_{i}, \mathbf{s}^{2} \cdot \beta=\sum_{j=1}^{d_{2}} s_{j}^{2} \beta_{j}\right)$, where $k !=\Gamma(k+1), k \geq 0$. Then $G^{(\mathbf{s})}(R)$ and $\tilde{G}^{\left(\mathbf{s}^{1}, \mathbf{s}^{2}\right)}\left(R^{1}, R^{2}\right)$ are Banach spaces equipped with the norms $\|\cdot\|_{R}^{(\mathbf{s})}$ and $\|\cdot \cdot\|_{R^{1}, R^{2}}^{\left(\mathbf{s}^{1}, \mathbf{s}^{2}\right)}$, respectively.

Similar definitions of Banach spaces of Gevrey type can be found in Miyake-Hashimoto [5].

Lemma 3.1. (i) If $s_{i} \geq 1$ for all $i=1, \ldots, d$, then

$$
G^{(\mathbf{s})}=\bigcup_{R \in\left(\mathbf{R}_{+} \backslash\{0\}\right)^{d}} G^{(\mathbf{s})}(R) .
$$

(ii) If $s_{i}^{1}, s_{j}^{2} \geq 1$ for all $i=1, \ldots, d_{1} ; j=1, \ldots, d_{2}$, then

$$
G^{\left(\mathbf{s}^{1}, \mathbf{s}^{2}\right)}=\bigcup_{\left(R^{1}, R^{2}\right) \in\left(\mathbf{R}_{+} \backslash\{0\}\right)^{d_{1}+d_{2}}} \tilde{G}^{\left(\mathbf{s}^{1}, \mathbf{s}^{2}\right)}\left(R^{1}, R^{2}\right) .
$$

Proof. (i): By Stirling's formula we can easily show that

$$
\alpha ! \leq|\alpha| ! \leq d^{|\alpha|} \alpha ! \quad \text { and } \quad A \cdot B^{|\alpha|} \leq \frac{(\alpha !)^{\mathbf{s}}}{(\mathbf{s} \cdot \alpha) !} \leq C \cdot D^{|\alpha|}
$$


for some constants $A, B, C$ and $D$, where $(\alpha !)^{\mathbf{s}}=\left(\alpha_{1} !\right)^{s_{1}} \ldots\left(\alpha_{d} !\right)^{s_{d}}$. This completes the proof.

(ii): Similar to (i).

Lemma 3.2. Assume that $a(x)=\sum_{\beta \in \mathbf{N}^{d}} a_{\beta} x^{\beta}$ and $a(x, y)=\sum_{(\gamma, \delta) \in \mathbf{N}^{d_{1}+d_{2}}}$. $a_{\gamma \delta} x^{\gamma} y^{\delta}$ are holomorphic functions on $\prod_{i=1}^{d}\left\{x_{i} \in \mathbf{C} ;\left|x_{i}\right| \leq T_{i}\right\}$ and $\prod_{i=1}^{d_{1}}\left\{x_{i} \in \mathbf{C}\right.$; $\left.\left|x_{i}\right| \leq T_{i}^{1}\right\} \times \prod_{j=1}^{d_{2}}\left\{y_{j} \in \mathbf{C} ;\left|y_{j}\right| \leq T_{j}^{2}\right\}\left(T=\left(T_{1}, \ldots, T_{d}\right) \in\left(\mathbf{R}_{+} \backslash\{0\}\right)^{d},\left(T^{1}, T^{2}\right)=\right.$ $\left.\left(T_{1}^{1}, \ldots, T_{d_{1}}^{1}, T_{1}^{2}, \ldots, T_{d_{2}}^{2}\right) \in\left(\mathbf{R}_{+} \backslash\{0\}\right)^{d_{1}+d_{2}}\right)$, respectively.

(i) If $0<R_{i} \leq T_{i}$ for all $i=1, \ldots, d$, the multiplication operator $a(x)$ is bounded on $G^{(\mathbf{s})}(R)$ for all $\mathbf{s}\left(\forall s_{i} \geq 1\right)$ with the norm bounded by $\sum_{\beta \in \mathbf{N}^{d}}\left|a_{\beta}\right| T^{\beta}$.

(ii) If $0<R_{i}^{1} \leq T_{i}^{1}$ and $0<R_{j}^{2} \leq T_{j}^{2}$ for all $i=1, \ldots, d_{1} ; j=1, \ldots, d_{2}$, the multiplication operator $a(x, y)$ is bounded on $\tilde{G}^{\left(\mathbf{s}^{1}, \mathbf{s}^{2}\right)}\left(R^{1}, R^{2}\right)$ for all $\left(\mathbf{s}^{1}, \mathbf{s}^{2}\right)$ $\left(\forall s_{i}^{1}, \forall s_{j}^{2} \geq 1\right)$ with the norm bounded by $\sum_{(\gamma, \delta) \in \mathbf{N}^{d_{1}+d_{2}}}\left|a_{\gamma \delta}\right|\left(T^{1}\right)^{\gamma}\left(T^{2}\right)^{\delta}$.

Proof. (i) is easily proved by the inequality

$$
\frac{|\alpha+\beta| !}{|\alpha| !}=\prod_{k=1}^{|\beta|}(|\alpha|+k) \leq \prod_{k=1}^{|\beta|}(\mathbf{s} \cdot \alpha+k)=\frac{(\mathbf{s} \cdot \alpha+|\beta|) !}{(\mathbf{s} \cdot \alpha) !} \leq \frac{(\mathbf{s} \cdot(\alpha+\beta)) !}{(\mathbf{s} \cdot \alpha) !}
$$

Similarly we can prove (ii) if we note that

$$
\frac{|\alpha+\gamma| !|\beta+\delta| !}{|\alpha| !|\beta| !} \leq \frac{\left(\mathbf{s}^{1} \cdot(\alpha+\gamma)+\mathbf{s}^{2} \cdot(\beta+\delta)\right) !}{\left(\mathbf{s}^{1} \cdot \alpha+\mathbf{s}^{2} \cdot \beta\right) !}
$$

\section{§4. Proof of Theorem 2.1 (when $m=0$ )}

In this section we shall prove Theorem 2.1 when $m=0$ (i.e., the cases (v), (vi) and (vii)). As mentioned in Section 2 we only consider the case (vi). Furthermore, for simplicity we assume $k=1$. We denote a formal power series solution as $u(y)=\sum_{\alpha \in \mathbf{N}^{d}} u_{\alpha} y^{\alpha}$ and use the Banach space $G^{(\boldsymbol{\sigma})}(Y)$ instead of $G^{(\mathbf{s})}(R)$. Therefore $u(y) \in G^{(\sigma)}(Y)$ means

$$
\|u\|_{Y}^{(\boldsymbol{\sigma})} \equiv \sum_{\alpha \in \mathbf{N}^{d}}\left|u_{\alpha}\right| \frac{|\alpha| !}{(\boldsymbol{\sigma} \cdot \alpha) !} Y^{\alpha}<\infty .
$$

We recall that the operator $P_{1}$ has the following form: $P_{1}=b(0)+b_{1}+$ $P_{1}^{\prime \prime \prime}$, where

$$
\begin{aligned}
P_{1}^{\prime \prime \prime} & =\sum_{j=1}^{d-1} y_{j+1} \frac{\partial}{\partial y_{j}}+\sum_{j=1}^{d}\left(\sum_{|\beta| \geq 2}^{\text {finite }} d_{j \beta}(y) y^{\beta}\right) \frac{\partial}{\partial y_{j}} \\
b_{1} & =b_{1}(y)=\sum_{|\beta| \geq 1}^{\text {finite }} b_{\beta}(y) y^{\beta} .
\end{aligned}
$$


Our problem is the unique solvability in $G^{(\sigma)}$ of the equation

$$
P_{1} U(y)=f(y)
$$

with $f(y) \in G^{(\boldsymbol{\sigma})}$.

We assume that $\sigma$ satisfies the condition (vi) in Theorem 2.1.

Proof of the case (vi) of Theorem 2.1. We may assume $b(0)=1$ since $b(0) \neq 0$. Next we estimate the operator norm of $b_{1}$ and $P_{1}^{\prime \prime \prime}$ on the space $G^{(\sigma)}(Y)$.

By Lemma 3.2, it holds that $b_{1}: G^{(\sigma)}(Y) \rightarrow G^{(\sigma)}(Y)$ is bounded for sufficiently small $Y$ with the estimate

$$
\left\|b_{1} U\right\|_{Y}^{(\sigma)} \leq C\left\{\sum_{i \beta \mid \geq 1}^{\text {finite }} Y^{\beta}\right\}\|U\|_{Y}^{(\sigma)}
$$

for some constant $C$. Here and hereafter $Y=\left(Y_{1}, \ldots, Y_{d}\right)$ is taken so small such that the coefficients of the operators $\partial / \partial y_{j}$, etc., and a function $f(y)$ belong to $G^{(\sigma)}(Y)$. In order to estimate the operator norm of $P_{1}^{\prime \prime \prime}$ we need the following:

Lemma 4.1. Let $\sigma, \beta$, and $\beta^{\prime}$ satisfy

$$
\sigma_{i} \geq 1(\forall i=1, \ldots, d) \quad \text { and } \quad \sigma \cdot\left(\beta-\beta^{\prime}\right) \geq|\beta| .
$$

Then the operator $y^{\beta} D_{y}^{\beta^{\prime}}$ is bounded on $G^{(\sigma)}(Y)$ and the operator norm is bounded by $Y^{\beta} / Y^{\beta^{\prime}}$.

Remark 1. Put

$$
\begin{aligned}
N\left(y^{\beta} D_{y}^{\beta^{\prime}}\right) & =\left\{(\mathscr{Y}, \mathscr{W}) \in \mathbb{R}^{d+1} ; \mathscr{Y}_{j} \geq \beta_{j}-\beta_{j}^{\prime}, \mathscr{W} \leq\left|\beta^{\prime}\right|\right\} \\
\Lambda(\sigma) & =\left\{(\mathscr{Y}, \mathscr{W}) \in \mathbb{R}^{d+1} ;(\sigma-\mathbb{1}) \cdot \mathscr{Y}-\mathscr{W} \geq 0\right\}
\end{aligned}
$$

and

$$
S=\left\{\boldsymbol{\sigma} ; N\left(y^{\beta} D_{y}^{\beta^{\prime}}\right) \subset \Lambda(\boldsymbol{\sigma})\right\} .
$$

Then the condition $\sigma \in S$ is equivalent to (4.2).

Proof of Lemma 4.1. For $U(y)=\sum_{\alpha \in \mathbf{N}^{d}} U_{\alpha} y^{\alpha} \in G^{(\boldsymbol{\sigma})}(Y)$, we have

$$
y^{\beta} D_{y}^{\beta^{\prime}} U(y)=\sum_{\alpha \geq \beta^{\prime}} \frac{\alpha !}{\left(\alpha-\beta^{\prime}\right) !} U_{\alpha} y^{\alpha+\beta-\beta^{\prime}} \text {. }
$$

Here $\alpha \geq \beta^{\prime}$ means that $\alpha_{i} \geq \beta_{i}^{\prime}$ for all $i$. Thus we have

$$
\left\|y^{\beta} D_{y}^{\beta^{\prime}} U\right\|_{Y}^{(\boldsymbol{\sigma})}=\sum_{\alpha \geq \beta^{\prime}}\left|\frac{\alpha !}{\left(\alpha-\beta^{\prime}\right) !} U_{\alpha}\right| \frac{\left|\alpha+\beta-\beta^{\prime}\right| !}{\left(\boldsymbol{\sigma} \cdot\left(\alpha+\beta-\beta^{\prime}\right)\right) !} Y^{\alpha+\beta-\beta^{\prime}} \equiv(A) .
$$


Furthermore, by (4.2) it holds that

$$
\frac{\alpha !}{\left(\alpha-\beta^{\prime}\right) !} \cdot \frac{\left|\alpha+\beta-\beta^{\prime}\right| !}{\left(\sigma \cdot\left(\alpha+\beta-\beta^{\prime}\right)\right) !} \leq \frac{|\alpha| !}{(\sigma \cdot \alpha) !} .
$$

Therefore we obtain

$$
(\boldsymbol{A}) \leq \frac{Y^{\beta}}{Y^{\beta^{\prime}}} \sum_{\alpha \geq \beta^{\prime}} \frac{|\alpha| !}{(\boldsymbol{\sigma} \cdot \alpha) !}\left|U_{\alpha}\right| Y^{\alpha} \leq \frac{Y^{\beta}}{Y^{\beta^{\prime}}}\|U\|_{Y}^{(\boldsymbol{\sigma})} .
$$

The proof is completed.

Proof of the case (vi) of Theorem 2.1 (continued). By the assumption $\sigma \in S_{6}$, Lemma 3.2 and 4.1 , it holds that $P_{1}^{\prime \prime \prime}: G^{(\sigma)}(Y) \rightarrow G^{(\sigma)}(Y)$ is bounded for sufficiently small $Y$ and that

$$
\left\|P_{1}^{\prime \prime \prime} U\right\|_{Y}^{(\boldsymbol{\sigma})} \leq C\left\{\sum_{j=1}^{d-1} \frac{Y_{j+1}}{Y_{j}}+\sum_{j=1}^{d}\left(\sum_{|\beta| \geq 2}^{\text {finite }} Y^{\beta}\right) \frac{1}{Y_{j}}\right\}
$$

Therefore for sufficiently small $Y$ the operator $b_{1}+P_{1}^{\prime \prime \prime}: G^{(\sigma)}(Y) \rightarrow G^{(\sigma)}(Y)$ is bounded with the estimate

$$
\left\|b_{1}+P_{1}^{\prime \prime \prime}\right\| \leq C\left\{\sum_{|\beta| \geq 1}^{\text {finite }} Y^{\beta}+\sum_{j=1}^{d-1} \frac{Y_{j+1}}{Y_{j}}+\sum_{j=1}^{d}\left(\sum_{|\beta| \geq 2}^{\text {finite }} Y^{\beta}\right) \frac{1}{Y_{j}}\right\} \equiv K,
$$

for some constant $C$. The constant $K$ can be taken arbitrarily small by an appropriate choice of $Y$. Hence, by taking $Y$ so that $K<1$ the operator $T$ defined by

$$
T U(y)=-\left(b_{1}+P_{1}^{\prime \prime \prime}\right) U(y)+f(y)
$$

is a contraction mapping on $G^{(\sigma)}(Y)$ in the sense of $\|T U-T V\| \leq K\|U-V\|$, because $f(y) \in G^{(\boldsymbol{\sigma})}(Y)$. Hence there exists a unique $U(y) \in G^{(\boldsymbol{\sigma})}(Y)$ which satisfies $T U(y)=U(y)$. Thus $U(y) \in G^{(\sigma)}$ is a solution of (4.1) by Lemma 3.1. This completes the proof.

Remark 2. The cases (v) and (vii) are proved similarly as the case (vi). Here we only refer to Banach spaces employed in the proof.

(v) Let us assume $k=1$. We use the Banach space $G^{((\sigma, \tau))}((Y, Z))$ whose elements $u(y, z)=\sum_{(\alpha, \beta) \in \mathbf{N}^{n+p}} u_{\alpha \beta} y^{\alpha} z^{\beta}(n+p=d)$ satisfy

$$
\|u\|_{(Y, Z)}^{((\boldsymbol{\sigma}, \boldsymbol{\tau}))} \equiv \sum_{(\alpha, \beta) \in \mathbf{N}^{n+p}}\left|u_{\alpha \beta}\right| \frac{(|\alpha|+|\beta|) !}{(\boldsymbol{\sigma} \cdot \alpha+\boldsymbol{\tau} \cdot \beta) !} Y^{\alpha} Z^{\beta}<\infty .
$$

(vii) We use the Banach space $G^{(\tau)}(Z)$ whose elements $u(z)=\sum_{\alpha \in \mathbf{N}^{d}} u_{\alpha} z^{\alpha}$ 
satisfy

$$
\|u\|_{Z}^{(\tau)} \equiv \sum_{\alpha \in \mathbf{N}^{d}}\left|u_{\alpha}\right| \frac{|\alpha| !}{(\tau \cdot \alpha) !} Z^{\alpha}<\infty .
$$

Remark 3. In the above proof we considered the case $k=1$. In the general case $k \geq 2$, for the variables $\left(y_{1}^{h}, \ldots, y_{n_{h}}^{h}\right)(h=1, \ldots, k)$ corresponding to a nilpotent matrix $B_{h}$, we have a similar estimate as (4.3), which implies the conclusion.

\section{§5. Proof of Theorem 2.1 (when $m \geq 1$ )}

We shall prove Theorem 2.1 when $m \geq 1$ (i.e., the cases (i), (ii), (iii) and (iv)). We consider only the case (ii). By the same reason as in the previous section we consider the case $k=1$. We denote a formal power series as $u(x, y)$ $=\sum_{(\alpha, \beta) \in \mathbf{N}^{m+n}} u_{\alpha \beta} x^{\alpha} y^{\beta}(m+n=d)$ and use the Banach space $\tilde{G}^{(\boldsymbol{\rho}, \boldsymbol{\sigma})}(X, Y)$ (resp. $\left.G^{((\boldsymbol{\rho}, \boldsymbol{\sigma}))}((X, Y))\right)$ instead of $\tilde{G}^{\left(\mathbf{s}^{1}, \mathbf{s}^{2}\right)}\left(R^{1}, R^{2}\right) \quad\left(\right.$ resp. $\left.G^{\left(\left(\mathbf{s}^{1}, \mathbf{s}^{2}\right)\right)}\left(\left(R^{1}, R^{2}\right)\right)\right)$. Therefore $u(x, y) \in \tilde{G}^{(\boldsymbol{\rho}, \boldsymbol{\sigma})}(X, Y)$ (resp. $\left.G^{((\boldsymbol{\rho}, \boldsymbol{\sigma}))}((X, Y))\right)$ means

$$
\begin{gathered}
\|u\|_{X, Y}^{(\boldsymbol{\rho}, \boldsymbol{\sigma})} \equiv \sum_{(\alpha, \beta) \in \mathbf{N}^{m+n}}\left|u_{\alpha \beta}\right| \frac{|\alpha| !|\beta| !}{(\boldsymbol{\rho} \cdot \alpha+\boldsymbol{\sigma} \cdot \beta) !} X^{\alpha} Y^{\beta}<\infty \\
\text { resp. } \left.\|u\|_{(X, Y)}^{((\boldsymbol{\rho}, \boldsymbol{\sigma}))} \equiv \sum_{(\alpha, \beta) \in \mathbf{N}^{m+n}}\left|u_{\alpha \beta}\right| \frac{(|\alpha|+|\beta|) !}{(\boldsymbol{\rho} \cdot \alpha+\boldsymbol{\sigma} \cdot \beta) !} X^{\alpha} Y^{\beta}<\infty\right) .
\end{gathered}
$$

We recall that the operator $P_{1}$ has the following form: $P_{1}=\sum_{i=1}^{m}$. $\lambda_{i} x_{i}\left(\partial / \partial x_{i}\right)+b(0)+P_{1}^{\prime}+P_{1}^{\prime \prime}+P_{1}^{\prime \prime \prime}+P_{1}^{\prime \prime \prime \prime}$, where

$$
\begin{aligned}
P_{1}^{\prime} & =\sum_{i=1}^{m-1} \delta_{i} x_{i+1} \frac{\partial}{\partial x_{i}}+\sum_{i=1}^{m}\left(\sum_{|\alpha|+|\beta| \geq 2,|\alpha| \geq 1}^{\text {finite }} c_{i \alpha \beta}(x, y) x^{\alpha} y^{\beta}\right) \frac{\partial}{\partial x_{i}}, \\
P_{1}^{\prime \prime} & =\sum_{j=1}^{n}\left(\sum_{|\alpha|+|\beta| \geq 2,|\alpha| \geq 1}^{\text {finite }} d_{j \alpha \beta}(x, y) x^{\alpha} y^{\beta}\right) \frac{\partial}{\partial y_{j}}, \\
P_{1}^{\prime \prime \prime} & =\sum_{j=1}^{n-1} y_{j+1} \frac{\partial}{\partial y_{j}}+\sum_{j=1}^{n}\left(\sum_{|\beta| \geq 2}^{\text {finite }} d_{j \beta}(x, y) y^{\beta}\right) \frac{\partial}{\partial y_{j}}, \\
P_{1}^{\prime \prime \prime \prime} & =\sum_{i=1}^{m}\left(\sum_{|\beta| \geq 2}^{\text {finite }} c_{i \beta}(x, y) y^{\beta}\right) \frac{\partial}{\partial x_{i}}, \\
b_{1} & =b_{1}(x, y)=\sum_{|\alpha|+|\beta| \geq 1}^{\text {finite }} b_{\alpha \beta}(x, y) x^{\alpha} y^{\beta} .
\end{aligned}
$$

Our problem is the unique solvability in $G^{(\rho, \sigma)}$ of the equation 


$$
P_{1} u(x, y)=f(x, y)
$$

with $f(x, y) \in G^{(\boldsymbol{\rho}, \boldsymbol{\sigma})}$.

Now we assume that $(\boldsymbol{\rho}, \boldsymbol{\sigma})$ satisfies the condition (ii) in Theorem 2.1.

Proof of the case (ii) of Theorem 2.1. First we define the operator $\Lambda_{b}: G^{(\boldsymbol{\rho}, \boldsymbol{\sigma})} \rightarrow G^{(\boldsymbol{\rho}, \boldsymbol{\sigma})}$ by

$$
\Lambda_{b}=\sum_{i=1}^{m} \lambda_{i} x_{i} \frac{\partial}{\partial x_{i}}+b(0)
$$

Then by the condition (Po) the operator $\Lambda_{b}$ is bijective and $\Lambda_{b}^{-1}$ is given by

$$
\Lambda_{b}^{-1} \sum_{(\alpha, \beta) \in \mathbf{N}^{m+n}} u_{\alpha \beta} x^{\alpha} y^{\beta}=\sum_{(\alpha, \beta) \in \mathbf{N}^{m+n}} \frac{u_{\alpha \beta}}{\lambda \cdot \alpha+b(0)} x^{\alpha} y^{\beta},
$$

where $\lambda \cdot \alpha=\sum_{i=1}^{m} \lambda_{i} \alpha_{i}$.

Keeping in mind the above expression we introduce a new unknown function $U(x, y)$ by

$$
U(x, y)=\Lambda_{b} u(x, y), \quad \text { that is, } u(x, y)=\Lambda_{b}^{-1} U(x, y) .
$$

Then the equation (5.1) is equivalent to the following one:

$$
\begin{aligned}
U(x, y) & +b_{2} U(x, y)+P_{2}^{\prime} U(x, y)+P_{2}^{\prime \prime} U(x, y) \\
& +P_{2}^{\prime \prime \prime} U(x, y)+P_{2}^{\prime \prime \prime \prime} U(x, y)=f(x, y)
\end{aligned}
$$

where

$$
\begin{aligned}
P_{2}^{\prime} U(x, y)= & \sum_{i=1}^{m-1} \delta_{i} x_{i+1} \frac{\partial}{\partial x_{i}} \Lambda_{b}^{-1} U(x, y) \\
& +\sum_{i=1}^{m}\left(\sum_{|\alpha|+|\beta| \geq 2,|\alpha| \geq 1}^{\text {finite }} c_{i \alpha \beta}(x, y) x^{\alpha} y^{\beta}\right) \frac{\partial}{\partial x_{i}} \Lambda_{b}^{-1} U(x, y) \\
P_{2}^{\prime \prime} U(x, y)= & \sum_{j=1}^{n}\left(\sum_{|\alpha|+|\beta| \geq 2,|\alpha| \geq 1}^{\text {finite }} d_{j \alpha \beta}(x, y) x^{\alpha} y^{\beta}\right) \frac{\partial}{\partial y_{j}} \Lambda_{b}^{-1} U(x, y) \\
P_{2}^{\prime \prime \prime} U(x, y)= & \sum_{j=1}^{n-1} y_{j+1} \frac{\partial}{\partial y_{j}} \Lambda_{b}^{-1} U(x, y)+\sum_{j=1}^{n}\left(\sum_{|\beta| \geq 2}^{\text {finite }} d_{j \beta}(x, y) y^{\beta}\right) \frac{\partial}{\partial y_{j}} \Lambda_{b}^{-1} U(x, y), \\
P_{2}^{\prime \prime \prime \prime} U(x, y)= & \sum_{i=1}^{m}\left(\sum_{|\beta| \geq 2}^{\text {finite }} c_{i \beta}(x, y) y^{\beta}\right) \frac{\partial}{\partial x_{i}} \Lambda_{b}^{-1} U(x, y) \\
b_{2} U(x, y)= & \sum_{|\alpha|+|\beta| \geq 1}^{\text {finite }} b_{\alpha \beta}(x, y) x^{\alpha} y^{\beta} \Lambda_{b}^{-1} U(x, y) .
\end{aligned}
$$


Next we estimate the operator norm of $b_{2}+P_{2}^{\prime}+P_{2}^{\prime \prime}+P_{2}^{\prime \prime \prime}+P_{2}^{\prime \prime \prime \prime}$ on the space $G(X, Y)$, where

$$
G(X, Y)= \begin{cases}\tilde{G}^{(\boldsymbol{\rho}, \boldsymbol{\sigma})}(X, Y) & \text { if } \left.P_{2}^{\prime \prime} \neq 0, \text { that is, } P_{1}^{\prime \prime} \neq 0\right) \\ G^{((\boldsymbol{\rho}, \boldsymbol{\sigma}))}((X, Y)) & \left(\text { if } P_{2}^{\prime \prime}=0, \text { that is, } P_{1}^{\prime \prime}=0\right) .\end{cases}
$$

Hereafter $X$ and $Y$ are taken so small that the coefficients of the operators and a function $f(x, y)$ belong to $G(X, Y)$.

Since $|1 /(\lambda \cdot \alpha+b(0))| \leq C$ for some constant $C$, the operator $\Lambda_{b}^{-1}: \tilde{G}^{(\boldsymbol{\rho}, \boldsymbol{\sigma})}(X, Y) \rightarrow \tilde{G}^{(\boldsymbol{\rho}, \boldsymbol{\sigma})}(X, Y) \quad\left(\operatorname{resp} . G^{((\boldsymbol{\rho}, \boldsymbol{\sigma}))}((X, Y)) \rightarrow G^{((\boldsymbol{\rho}, \boldsymbol{\sigma}))}((X, Y))\right)$ is bounded and we have

$$
\left.\left\|\Lambda_{b}^{-1} U\right\|_{X, Y}^{(\boldsymbol{\rho}, \boldsymbol{\sigma})} \leq C\|U\|_{X, Y}^{(\boldsymbol{\rho}, \boldsymbol{\sigma})} \quad \text { (resp. }\left\|\Lambda_{b}^{-1} U\right\|_{(X, Y)}^{((\boldsymbol{\rho}, \boldsymbol{\sigma}))} \leq C\|U\|_{(X, Y)}^{((\boldsymbol{\rho}, \boldsymbol{\sigma}))}\right) .
$$

Therefore by Lemma 3.2, $b_{2}: \tilde{G}^{(\boldsymbol{\rho}, \boldsymbol{\sigma})}(X, Y) \rightarrow \tilde{G}^{(\boldsymbol{\rho}, \boldsymbol{\sigma})}(X, Y)\left(\operatorname{resp} . G^{((\boldsymbol{\rho}, \boldsymbol{\sigma}))}((X, Y))\right.$ $\left.\rightarrow G^{((\boldsymbol{\rho}, \boldsymbol{\sigma}))}((X, Y))\right)$ is bounded and we have

$$
\begin{gathered}
\left\|b_{2} U\right\|_{X, Y}^{(\boldsymbol{\rho}, \boldsymbol{\sigma})} \leq C\left(\sum_{|\alpha|+|\beta| \geq 1}^{\text {finite }} X^{\alpha} Y^{\beta}\right)\|U\|_{X, Y}^{(\boldsymbol{\rho}, \boldsymbol{\sigma})} \\
\left(\text { resp. }\left\|b_{2} U\right\|_{(X, Y)}^{(\boldsymbol{\rho}, \boldsymbol{\sigma}))} \leq C\left(\sum_{|\alpha|+|\beta| \geq 1}^{\text {finite }} X^{\alpha} Y^{\beta}\right)\|U\|_{(X, Y)}^{((\boldsymbol{\rho}, \boldsymbol{\sigma}))}\right) .
\end{gathered}
$$

In order to estimate the operator norm of $P_{2}^{\prime}+P_{2}^{\prime \prime}+P_{2}^{\prime \prime \prime}+P_{2}^{\prime \prime \prime \prime}$ we need the following lemma:

Lemma 5.1. (i) Let $\boldsymbol{\rho}, \boldsymbol{\sigma}, \gamma, \delta, \gamma^{\prime}$ and $\delta^{\prime}$ satisfy

$$
\rho_{i}, \sigma_{j} \geq 1(\forall i, j) \quad \text { and } \quad \boldsymbol{\rho} \cdot\left(\gamma-\gamma^{\prime}\right)+\sigma \cdot\left(\delta-\delta^{\prime}\right) \geq|\gamma|+|\delta| .
$$

Then the operator $x^{\gamma} y^{\delta} D_{x}^{\gamma^{\prime}} D_{y}^{\delta^{\prime}} \Lambda_{b}^{-1}$ is bounded on $\tilde{G}^{(\boldsymbol{\rho}, \boldsymbol{\sigma})}(X, Y)$ and $G^{((\boldsymbol{\rho}, \boldsymbol{\sigma}))}((X, Y))$, and the operator norm is bounded by $C X^{\gamma} Y^{\delta} / X^{\gamma^{\prime}} Y^{\delta^{\prime}}$.

(ii) If $|\gamma| \geq 1$,

$$
\rho_{i}, \sigma_{j} \geq 1(\forall i, j) \quad \text { and } \quad \boldsymbol{\rho} \cdot\left(\gamma-\gamma^{\prime}\right)+\sigma \cdot\left(\delta-\delta^{\prime}\right) \geq|\gamma|+|\delta|-1,
$$

then the operator $x^{\gamma} y^{\delta} D_{x}^{\gamma^{\prime}} D_{y}^{\delta^{\prime}} \Lambda_{b}^{-1}$ is bounded on $\tilde{G}^{(\rho, \sigma)}(X, Y)$, and the operator norm is bounded by $C_{\gamma \gamma^{\prime}} X^{\gamma} Y^{\delta} / X^{\gamma^{\prime}} Y^{\delta^{\prime}}$.

(iii) If $\left|\gamma^{\prime}\right| \geq 1$ and (5.4) hold, then the operator $x^{\gamma} y^{\delta} D_{x}^{\gamma^{\prime}} D_{y}^{\delta^{\prime}} \Lambda_{b}^{-1}$ is bounded on $G^{((\boldsymbol{\rho}, \boldsymbol{\sigma}))}((X, Y))$, and the operator norm is bounded by $C_{\gamma \delta \gamma^{\prime} \delta^{\prime}} X^{\gamma} Y^{\delta} / X^{\gamma^{\prime}} Y^{\delta^{\prime}}$.

Remark 1. Put

$$
\begin{aligned}
N\left(x^{\gamma} y^{\delta} D_{x}^{\gamma^{\prime}} D_{y}^{\delta^{\prime}}\right) & =\left\{(\mathscr{X}, \mathscr{Y}, \mathscr{W}) \in \mathbb{R}^{d+1} ; \mathscr{X}_{i} \geq \gamma_{i}-\gamma_{i}^{\prime}, \mathscr{Y}_{j} \geq \delta_{j}-\delta_{j}^{\prime}, \mathscr{W} \leq\left|\gamma^{\prime}\right|+\left|\delta^{\prime}\right|\right\} \\
\tilde{\Lambda}(\boldsymbol{\rho}, \boldsymbol{\sigma}) & =\left\{(\mathscr{X}, \mathscr{Y}, \mathscr{W}) \in \mathbb{R}^{d+1} ;(\boldsymbol{\rho}-\mathbb{1}) \cdot \mathscr{X}+(\boldsymbol{\sigma}-\mathbb{1}) \cdot \mathscr{Y}-\mathscr{W} \geq-1\right\} \\
\Lambda(\boldsymbol{\rho}, \boldsymbol{\sigma}) & =\left\{(\mathscr{X}, \mathscr{Y}, \mathscr{W}) \in \mathbb{R}^{d+1} ;(\boldsymbol{\rho}-\mathbb{1}) \cdot \mathscr{X}+(\boldsymbol{\sigma}-\mathbb{1}) \cdot \mathscr{Y}-\mathscr{W} \geq 0\right\}
\end{aligned}
$$


and set

$$
\begin{aligned}
& \tilde{S}=\left\{(\boldsymbol{\rho}, \boldsymbol{\sigma}) ; N\left(x^{\gamma} y^{\delta} D_{x}^{\gamma^{\prime}} D_{y}^{\delta^{\prime}}\right) \subset \tilde{\Lambda}(\boldsymbol{\rho}, \boldsymbol{\sigma})\right\} \\
& S=\left\{(\boldsymbol{\rho}, \boldsymbol{\sigma}) ; N\left(x^{\gamma} y^{\delta} D_{x}^{\gamma^{\prime}} D_{y}^{\delta^{\prime}}\right) \subset \Lambda(\boldsymbol{\rho}, \boldsymbol{\sigma})\right\} .
\end{aligned}
$$

Then the conditions $(\boldsymbol{\rho}, \boldsymbol{\sigma}) \in \tilde{S}$ and $(\boldsymbol{\rho}, \boldsymbol{\sigma}) \in S$ are equivalent to (5.4) and (5.3), respectively.

Remark 2. In Lemma 5.1, (ii) does not hold on the space $G^{((\boldsymbol{\rho}, \boldsymbol{\sigma}))}((X, Y))$. On the other hand, (iii) does not hold on the space $\tilde{G}^{(\boldsymbol{\rho}, \boldsymbol{\sigma})}(X, Y)$. Therefore the operator $y^{\delta} D_{x}^{\gamma^{\prime}} D_{y}^{\delta^{\prime}} \Lambda_{b}^{-1}+x^{\gamma} y^{\delta} D_{y}^{\delta^{\prime}} \Lambda_{b}^{-1}\left(|\gamma|,\left|\gamma^{\prime}\right| \geq 1\right)$ is bounded neither on $G^{((\boldsymbol{\rho}, \boldsymbol{\sigma}))}((X, Y))$ nor on $\tilde{G}^{(\boldsymbol{\rho}, \boldsymbol{\sigma})}(X, Y)$, even if (5.4) holds (cf. the example in Remark 1 of Section 2).

Proof of Lemma 5.1. Because $U(x, y)=\sum_{(\alpha, \beta) \in \mathbf{N}^{m+n}} U_{\alpha \beta} x^{\alpha} y^{\beta}$ satisfies $x^{\gamma} y^{\delta} D_{x}^{\gamma^{\prime}} D_{y}^{\delta^{\prime}} \Lambda_{b}^{-1} U(x, y)=\sum_{\alpha \geq \gamma^{\prime}, \beta \geq \delta^{\prime}} \frac{1}{\lambda \cdot \alpha+b(0)} \frac{\alpha !}{\left(\alpha-\gamma^{\prime}\right) !} \frac{\beta !}{\left(\beta-\delta^{\prime}\right) !} U_{\alpha \beta} x^{\alpha+\gamma-\gamma^{\prime}} y^{\beta+\delta-\delta^{\prime}}$

we have

$$
\begin{aligned}
\left\|\mid x^{\gamma} y^{\delta} D_{x}^{\gamma^{\prime}} D_{y}^{\delta^{\prime}} \Lambda_{b}^{-1} U\right\|_{X, Y}^{(\boldsymbol{\rho}, \boldsymbol{\sigma})}= & \sum_{\alpha \geq \gamma^{\prime}, \beta \geq \delta^{\prime}} \frac{\left|U_{\alpha \beta}\right|}{|\lambda \cdot \alpha+b(0)|} \frac{\alpha !}{\left(\alpha-\gamma^{\prime}\right) !} \frac{\beta !}{\left(\beta-\delta^{\prime}\right) !} \\
& \times \frac{\left|\alpha+\gamma-\gamma^{\prime}\right| !\left|\beta+\delta-\delta^{\prime}\right| !}{\left(\boldsymbol{\rho} \cdot\left(\alpha+\gamma-\gamma^{\prime}\right)+\sigma \cdot\left(\beta+\delta-\delta^{\prime}\right)\right) !} X^{\alpha+\gamma-\gamma^{\prime}} Y^{\beta+\delta-\delta^{\prime}} .
\end{aligned}
$$

Similarly we have

$$
\begin{aligned}
\left\|x^{\gamma} y^{\delta} D_{x}^{\gamma^{\prime}} D_{y}^{\delta^{\prime}} \Lambda_{b}^{-1} U\right\|_{(X, Y)}^{(\boldsymbol{\rho}, \boldsymbol{\sigma}))}= & \sum_{\alpha \geq \gamma^{\prime}, \beta \geq \delta^{\prime}} \frac{\left|U_{\alpha \beta}\right|}{|\lambda \cdot \alpha+b(0)|} \frac{\alpha !}{\left(\alpha-\gamma^{\prime}\right) !} \frac{\beta !}{\left(\beta-\delta^{\prime}\right) !} \\
& \times \frac{\left(\left|\alpha+\gamma-\gamma^{\prime}\right|+\left|\beta+\delta-\delta^{\prime}\right|\right) !}{\left(\boldsymbol{\rho} \cdot\left(\alpha+\gamma-\gamma^{\prime}\right)+\boldsymbol{\sigma} \cdot\left(\beta+\delta-\delta^{\prime}\right)\right) !} X^{\alpha+\gamma-\gamma^{\prime}} Y^{\beta+\delta-\delta^{\prime}} .
\end{aligned}
$$

The case (i) is proved by the following inequalities: $1 /|\lambda \cdot \alpha+b(0)| \leq C$,

$$
\begin{aligned}
& \frac{\alpha !}{\left(\alpha-\gamma^{\prime}\right) !} \frac{\beta !}{\left(\beta-\delta^{\prime}\right) !} \frac{\left|\alpha+\gamma-\gamma^{\prime}\right| !\left|\beta+\delta-\delta^{\prime}\right| !}{\left(\boldsymbol{\rho} \cdot\left(\alpha+\gamma-\gamma^{\prime}\right)+\boldsymbol{\sigma} \cdot\left(\beta+\delta-\delta^{\prime}\right)\right) !} \leq \frac{|\alpha| !|\beta| !}{(\boldsymbol{\rho} \cdot \alpha+\boldsymbol{\sigma} \cdot \beta) !}, \\
& \frac{\alpha !}{\left(\alpha-\gamma^{\prime}\right) !} \frac{\beta !}{\left(\beta-\delta^{\prime}\right) !} \frac{\left(\left|\alpha+\gamma-\gamma^{\prime}\right|+\left|\beta+\delta-\delta^{\prime}\right|\right) !}{\left(\boldsymbol{\rho} \cdot\left(\alpha+\gamma-\gamma^{\prime}\right)+\boldsymbol{\sigma} \cdot\left(\beta+\delta-\delta^{\prime}\right)\right) !} \leq \frac{(|\alpha|+|\beta|) !}{(\boldsymbol{\rho} \cdot \alpha+\boldsymbol{\sigma} \cdot \beta) !}
\end{aligned}
$$

which are obtained by the condition (Po) and the assumption (5.3). 
The case (ii) is proved by the inequality

$$
\begin{aligned}
& \frac{\left|\alpha+\gamma-\gamma^{\prime}\right|}{|\lambda \cdot \alpha+b(0)|} \frac{\alpha !}{\left(\alpha-\gamma^{\prime}\right) !} \frac{\beta !}{\left(\beta-\delta^{\prime}\right) !} \\
& \quad \times \frac{\left(\left|\alpha+\gamma-\gamma^{\prime}\right|-1\right) !\left|\beta+\delta-\delta^{\prime}\right| !}{\left(\boldsymbol{\rho} \cdot\left(\alpha+\gamma-\gamma^{\prime}\right)+\sigma \cdot\left(\beta+\delta-\delta^{\prime}\right)\right) !} \leq C_{\gamma \gamma^{\prime}} \frac{(|\alpha| !|\beta| !)}{(\boldsymbol{\rho} \cdot \alpha+\boldsymbol{\sigma} \cdot \beta) !}
\end{aligned}
$$

which is obtained by the condition (Po) and the assumption (5.4). Note that $\left|\alpha+\gamma-\gamma^{\prime}\right|-1 \geq 0$ since $|\gamma| \geq 1$.

The case (iii) is proved by the inequality

$$
\begin{aligned}
& \frac{|\alpha|}{|\lambda \cdot \alpha+b(0)|} \frac{\left|\alpha+\gamma-\gamma^{\prime}\right|+\left|\beta+\delta-\delta^{\prime}\right|}{|\alpha|+|\beta|} \frac{(|\alpha|-1) !}{\left|\alpha-\gamma^{\prime}\right| !} \frac{|\beta| !}{\left|\beta-\delta^{\prime}\right| !} \\
& \quad \times \frac{\left(\left|\alpha+\gamma-\gamma^{\prime}\right|+\left|\beta+\delta-\delta^{\prime}\right|-1\right) !}{\left(\boldsymbol{\rho} \cdot\left(\alpha+\gamma-\gamma^{\prime}\right)+\sigma \cdot\left(\beta+\delta-\delta^{\prime}\right)\right) !} \leq C_{\gamma \delta \gamma^{\prime} \delta^{\prime}} \frac{(|\alpha|+|\beta|-1) !}{(\boldsymbol{\rho} \cdot \alpha+\sigma \cdot \beta) !}
\end{aligned}
$$

which is obtained by the condition (Po) and the assumption (5.4). Note that $|\alpha|-1 \geq 0$ since $|\alpha| \geq\left|\gamma^{\prime}\right| \geq 1$.

Proof of the case (ii) of Theorem 2.1 (continued). When $P_{2}^{\prime \prime \prime \prime}=0$ (that is, $P_{1}^{\prime \prime \prime \prime}=0$ ), it follows from the assumption $(\boldsymbol{\rho}, \sigma) \in \tilde{S}_{2} \cap S_{2}$, Lemma 3.2, (i) and (ii) of Lemma 5.1 that the map $b_{2}+P_{2}^{\prime}+P_{2}^{\prime \prime}+P_{2}^{\prime \prime \prime}: \tilde{G}^{(\boldsymbol{\rho}, \boldsymbol{\sigma})}(X, Y) \rightarrow \tilde{G}^{(\boldsymbol{\rho}, \boldsymbol{\sigma})}(X, Y)$ is bounded. Moreover the operator norm is estimated as

$$
\begin{aligned}
& \left\|\left|b_{2}+P_{2}^{\prime}+P_{2}^{\prime \prime}+P_{2}^{\prime \prime \prime} \|\right|\right. \\
& \leq C\left\{\sum_{|\alpha|+|\beta| \geq 1}^{\text {finite }} X^{\alpha} Y^{\beta}+\sum_{i=1}^{m-1} \frac{X_{i+1}}{X_{i}}+\sum_{i=1}^{m}\left(\sum_{|\alpha|+|\beta| \geq 2,|\alpha| \geq 1}^{\text {finite }} X^{\alpha} Y^{\beta}\right) \frac{1}{X_{i}}\right. \\
& \left.+\sum_{j=1}^{n}\left(\sum_{|\alpha|+|\beta| \geq 2,|\alpha| \geq 1}^{\text {finite }} X^{\alpha} Y^{\beta}\right) \frac{1}{Y_{j}}+\sum_{j=1}^{n-1} \frac{Y_{j+1}}{Y_{j}}+\sum_{j=1}^{n}\left(\sum_{|\beta| \geq 2}^{\text {finite }} Y^{\beta}\right) \frac{1}{Y_{j}}\right\} \equiv K_{1} .
\end{aligned}
$$

When $P_{2}^{\prime \prime}=0$ (that is, $P_{1}^{\prime \prime}=0$ ), it follows from the assumption $(\boldsymbol{\rho}, \boldsymbol{\sigma}) \in$ $\tilde{S}_{2} \cap S_{2} \cap S_{2}^{\prime}$, Lemma 3.2, (i) and (iii) of Lemma 5.1 that the map $b_{2}+P_{2}^{\prime}+$ $P_{2}^{\prime \prime \prime}+P_{2}^{\prime \prime \prime \prime}: G^{((\boldsymbol{\rho}, \boldsymbol{\sigma}))}((X, Y)) \rightarrow G^{((\boldsymbol{\rho}, \boldsymbol{\sigma}))}((X, Y))$ is bounded. Moreover the op- 
erator norm is estimated as

$$
\begin{aligned}
\| b_{2}+ & P_{2}^{\prime}+P_{2}^{\prime \prime \prime}+P_{2}^{\prime \prime \prime \prime} \| \\
\leq & C\left\{\sum_{|\alpha|+|\beta| \geq 1}^{\text {finite }} X^{\alpha} Y^{\beta}+\sum_{i=1}^{m-1} \frac{X_{i+1}}{X_{i}}+\sum_{i=1}^{m}\left(\sum_{|\alpha|+|\beta| \geq 2,|\alpha| \geq 1}^{\text {finite }} X^{\alpha} Y^{\beta}\right) \frac{1}{X_{i}}\right. \\
& \left.+\sum_{j=1}^{n-1} \frac{Y_{j+1}}{Y_{j}}+\sum_{j=1}^{n}\left(\sum_{|\beta| \geq 2}^{\text {finite }} Y^{\beta}\right) \frac{1}{Y_{j}}+\sum_{i=1}^{m}\left(\sum_{|\beta| \geq 2}^{\text {finite }} Y^{\beta}\right) \frac{1}{X_{i}}\right\} \equiv K_{2} .
\end{aligned}
$$

When $P_{2}^{\prime \prime \prime \prime}, P_{2}^{\prime \prime} \neq 0$ (that is, $P_{1}^{\prime \prime \prime \prime}, P_{1}^{\prime \prime} \neq 0$ ), it follows from the assumption $(\boldsymbol{\rho}, \boldsymbol{\sigma}) \in \tilde{S}_{2} \cap S_{2} \cap S_{2}^{\prime \prime}$, Lemma 3.2, (i) and (ii) of Lemma 5.1 that the map $b_{2}+P_{2}^{\prime}+P_{2}^{\prime \prime}+P_{2}^{\prime \prime \prime}+P_{2}^{\prime \prime \prime \prime}: \tilde{G}^{(\boldsymbol{\rho}, \boldsymbol{\sigma})}(X, Y) \rightarrow \tilde{G}^{(\boldsymbol{\rho}, \boldsymbol{\sigma})}(X, Y)$ is bounded. Moreover the operator norm is estimated as

$$
\begin{aligned}
\| b_{2}+ & P_{2}^{\prime}+P_{2}^{\prime \prime}+P_{2}^{\prime \prime \prime}+P_{2}^{\prime \prime \prime \prime}|\|| \\
\leq & C\left\{\sum_{|\alpha|+|\beta| \geq 1}^{\text {finite }} X^{\alpha} Y^{\beta}+\sum_{i=1}^{m-1} \frac{X_{i+1}}{X_{i}}+\sum_{i=1}^{m}\left(\sum_{|\alpha|+|\beta| \geq 2,|\alpha| \geq 1}^{\text {finite }} X^{\alpha} Y^{\beta}\right) \frac{1}{X_{i}}\right. \\
& +\sum_{j=1}^{n}\left(\sum_{|\alpha|+|\beta| \geq 2,|\alpha| \geq 1}^{\text {finite }} X^{\alpha} Y^{\beta}\right) \frac{1}{Y_{j}}+\sum_{j=1}^{n-1} \frac{Y_{j+1}}{Y_{j}}+\sum_{j=1}^{n}\left(\sum_{|\beta| \geq 2}^{\text {finite }} Y^{\beta}\right) \frac{1}{Y_{j}} \\
& \left.+\sum_{i=1}^{m}\left(\sum_{|\beta| \geq 2}^{\text {finite }} Y^{\beta}\right) \frac{1}{X_{i}}\right\} \equiv K_{3} .
\end{aligned}
$$

Finally we notice that we can take $X$ and $Y$ so that $K_{i}<1 \quad(i=1,2,3)$, which assure that each operator becomes a contraction on $G(X, Y)$. This completes the proof.

Remark 3. The cases (i), (iii) and (iv) are proved similarly as the case (ii). Here we only refer to Banach space employed in the proof for each case.

(i) Let us assume $k=1$. When $P_{1}^{\prime \prime} \neq 0$, we use the Banach space $\tilde{G}^{(\boldsymbol{\rho},(\boldsymbol{\sigma}, \tau))}(X,(Y, Z))$ whose elements $u(x, y, z)=\sum_{(\alpha, \beta, \gamma) \in \mathbf{N}^{m+n+p}} u_{\alpha \beta \gamma} x^{\alpha} y^{\beta} z^{\gamma}$. $(m+n+p=d)$ satisfy

$$
\|\| u \|_{X,(Y, Z)}^{(\boldsymbol{\rho},(\boldsymbol{\sigma}, \boldsymbol{\tau}))} \equiv \sum_{(\alpha, \beta, \gamma) \in \mathbf{N}^{m+n+p}} \frac{|\alpha| !(|\beta|+|\gamma|) !}{(\boldsymbol{\rho} \cdot \alpha+\boldsymbol{\sigma} \cdot \beta+\boldsymbol{\tau} \cdot \gamma) !}\left|u_{\alpha \beta \gamma}\right| X^{\alpha} Y^{\beta} Z^{\gamma}<\infty .
$$


When $P_{1}^{\prime \prime}=0$, we use the Banach space $G^{((\boldsymbol{\rho}, \boldsymbol{\sigma}, \tau))}((X, Y, Z))$ whose elements $u(x, y, z)=\sum_{(\alpha, \beta, \gamma) \in \mathbf{N}^{m+n+p}} u_{\alpha \beta \gamma} x^{\alpha} y^{\beta} z^{\gamma}$ satisfy

$$
\|u\|_{(X, Y, Z)}^{((\boldsymbol{\rho}, \boldsymbol{\sigma}, \tau))} \equiv \sum_{(\alpha, \beta, \gamma) \in \mathbf{N}^{m+n+p}} \frac{(|\alpha|+|\beta|+|\gamma|) !}{(\boldsymbol{\rho} \cdot \alpha+\boldsymbol{\sigma} \cdot \beta+\tau \cdot \gamma) !}\left|u_{\alpha \beta \gamma}\right| X^{\alpha} Y^{\beta} Z^{\gamma}<\infty .
$$

(iii) When $P_{1}^{\prime \prime} \neq 0$, we use the Banach space $\tilde{G}^{(\boldsymbol{\rho}, \tau)}(X, Z)$ whose elements $u(x, z)=\sum_{(\alpha, \gamma) \in \mathbf{N}^{m+p}} u_{\alpha \gamma} x^{\alpha} z^{\gamma}(m+p=d)$ satisfy

$$
\|||\|_{X, Z}^{(\boldsymbol{\rho}, \boldsymbol{\tau})} \equiv \sum_{(\alpha, \gamma) \in \mathbf{N}^{m+p}} \frac{|\alpha| !|\gamma| !}{(\boldsymbol{\rho} \cdot \alpha+\boldsymbol{\tau} \cdot \gamma) !}\left|u_{\alpha \gamma}\right| X^{\alpha} Z^{\gamma}<\infty
$$

When $P_{1}^{\prime \prime}=0$, we use the Banach space $G^{((\rho, \tau))}((X, Z))$ whose elements $u(x, z)=\sum_{(\alpha, \gamma) \in \mathbf{N}^{m+p}} u_{\alpha \gamma} x^{\alpha} z^{\gamma}$ satisfy

$$
\|u\|_{(X, Z)}^{((\boldsymbol{\rho}, \boldsymbol{\tau}))} \equiv \sum_{(\alpha, \gamma) \in \mathbf{N}^{m+p}} \frac{(|\alpha|+|\gamma|) !}{(\boldsymbol{\rho} \cdot \alpha+\boldsymbol{\tau} \cdot \gamma) !}\left|u_{\alpha \gamma}\right| X^{\alpha} Z^{\gamma}<\infty
$$

(iv) We use the Banach space $G^{(\mathbf{1})}(X)$ whose elements $u(x)=\sum_{\alpha \in \mathbf{N}^{d}} u_{\alpha} x^{\alpha}$ satisfy

$$
\|u\|_{X}^{(\mathbf{1})} \equiv \sum_{\alpha \in \mathbf{N}^{d}}\left|u_{\alpha}\right| X^{\alpha}<\infty
$$

\section{References}

[1] Gérard, R. and Tahara, H., Singular Nonlinear Partial Differential Equations in Complex Domain, Vieweg, 1996.

[2] Hibino, M., Gevrey asymptotic expansion for singular first order linear partial differential equations of nilpotent type (in Japanese), Master Thesis, Graduate School of Mathematics, Nagoya University (1998).

[3] Kashiwara, M., Kawai, T. and Shöstrand, J., On a class of linear partial differential equations whose formal solutions always converge, Ark. Mat., 17 (1979), 83-91.

[4] Miyake, M., Newton polygons and formal Gevrey indices in the Cauchy-Goursat-Fuchs type equations, J. Math. Soc. Japan, 43 (1991), 303-330.

[5] Miyake, M. and Hashimoto, Y., Newton Polygons and Gevrey Indices for Linear Partial Differential Operators, Nagoya Math. J., 128 (1992), 15-47.

[6] Miyake, M. and Shirai, A., Convergence of Formal Solutions of First Order Singular Nonlinear Partial Differential Equations in Complex Domain, to appear.

[7] Miyake, M. and Yoshino, M., Fredholm property of partial differential operators of irregular singular type, Ark. Mat., 33 (1995), 323-341. 
[8] Oshima, T., On the Theorem of Cauchy-Kowalevski for First Order Linear Differential Equation with Degenerate Principal Symbol, Proc. Japan Acad., 49 (1973), 83-87.

[9] Yamazawa, H., Newton polyhedrons and a formal Gevrey space of double indices for linear partial differential operators, to appear. 
